[Vicino Oriente XVI (2012), pp. 97-129]

\title{
MONTE SIRAI 2005-2010. BILANCI E PROSPETTIVE
}

\author{
Michele Guirguis - Università degli Studi di Sassari*
}

The site of Mount Sirai, investigated since the early '60s, currently is a field-research for the study of the Phoenician and Punic presence in the south-western Sardinia between the $8^{\text {th }}$ and $2^{\text {nd }}$ centuries. B.C. In the case of Monte Sirai, the internal position is probably linked to the existence of privileged relations between the Nuragic elements present in the area and the inhabitants of oriental origin. This paper presents new data from the necropolis and from the recent excavations in the socalled Acropoli. The tombs offer several contexts of infant burials and of a pregnant woman.

During the last seasons of excavations (2005-2010) has been detected a large number of ritual activities and funerary practices; the presence of a cemetery used between the $6^{\text {th }}$ and the $5^{\text {th }}$ centuries B.C., of which was found the ustrinum area, is related to discovery of bodies semi-burnt. The most recent research in the Acropoli has confirmed the horizon of most ancient presence in the second half of $8^{\text {th }}$ century B.C. and the final abandonment at the end of the $2^{\text {nd }}$ century B.C. The various traces of metallurgical activities confirm, finally, the strategic exploitation of mines in the Sulcis region.

Keywords: ceramica fenicia e punica; ustrinum; tombe infantili; donna gravida; attività metallurgiche

\section{INTRODUZIONE}

Il giacimento archeologico di Monte Sirai si presta, secondo una strategia d'intervento adottata fin dalle prime ricerche estensive degli anni '60, alla sistematica esplorazione dei diversi nuclei di cui si compone l'insediamento. Gli scavi che si svolgono con regolarità sul sito sotto la direzione scientifica di Piero Bartoloni producono un aggiornamento costante della documentazione disponibile ${ }^{1}$. Il pianoro di Monte Sirai conserva testimonianze di frequentazione umana fin dalla preistoria (Neolitico ed Eneolitico), mentre durante l'Età del Bronzo e la prima Età del Ferro si registra un'antropizzazione indiziata dalla presenza di alcuni nuraghi monotorre che costeggiano il pianoro ${ }^{2}$ e dai numerosi centri satelliti che controllavano la regione (tra cui il complesso del nuraghe Sirai ubicato alla base del monte, a ridosso del suo fianco sud-orientale ${ }^{3}$.

La presenza fenicia a Monte Sirai, allo stato attuale delle conoscenze, rappresenta un caposaldo fondamentale per l'irraggiamento delle genti di origine orientale nel milieu socio-culturale delle comunità autoctone del Sulcis (fig. 1). Tra la prima metà dell’VIII e la seconda metà del VII sec. a.C. tutto il territorio sulcitano appare solcato da una rete capillare costituita dai centri di nuova fondazione ma anche dalle realtà preesistenti di

* Dipartimento di Storia; micheleguirguis@yahoo.it.

1 Ringrazio il Prof. Lorenzo Nigro per l'invito a prendere parte ai lavori congressuali della IX Giornata dedicata alla memoria di Antonia Ciasca; sono inoltre particolarmente riconoscente nei confronti del Prof. Piero Bartoloni, direttore scientifico delle indagini a Monte Sirai e Sulky, col quale ho l'onore di collaborare ormai da oltre un decennio; le ricerche a Sirai si svolgono in collaborazione con la Soprintendenza Archeologica per le province di Cagliari e Oristano e con il prezioso contributo offerto dalle Amministrazioni Comunali di Carbonia e di Sant’Antioco, dalla Portovesme s.r.l. e dalla Società Ati-Ifras Intini.

2 Finocchi 2005, 229-238.

3 Perra 2007. 
natura autoctona con questi in contatto. Alla fondazione di Sulky4, di $\operatorname{Inosim}^{5}$ e dell'ipotetico abitato di riferimento della necropoli di S. Giorgio di Portoscuso ${ }^{6}$, fa seguito in progresso di tempo la creazione di insediamenti stabili in posizione sub-costiera e interna, a Monte Sirai (Carbonia) e a Pani Loriga (Santadi).

Per affrontare tali problematiche storico-archeologiche il centro di Monte Sirai costituisce un terreno di ricerca particolarmente promettente, sebbene debbano sottolinearsi i limiti derivanti dalle difficoltà di "traduzione” del correlato archeologico che, di necessità, produce un continuo affinamento dei criteri interpretativi. In questa nota si cercherà di fare un breve bilancio sugli ultimi sei anni di attività nell'insediamento, sintetizzando le diverse fasi della ricerca e presentando parte della documentazione ancora inedita. Le indagini stratigrafiche, concentrate fin dal 2005 nelle aree di necropoli, dal 2010 interessano anche un vasto settore dell'abitato la cui esplorazione, seppure agli inizi, ha già consentito di raccogliere numerosi dati (fig. 2).

\section{LA NECROPOLI FENICIA E PUNICA}

Tra il 2005 e il 2010 nella necropoli di Sirai (fig. 3) sono state indagate in totale 96 sepolture $^{7}$ distribuite tra la fine del VII e la metà del IV sec. a.C., con una prevalenza delle attestazioni tra il VI e il V sec. a.C. Le pratiche funebri mostrano una netta variabilità anche sincronica, molto accentuata soprattutto tra la seconda metà del VI e la prima metà del V sec. a.C., nel periodo contrassegnato dal passaggio della Sardegna sotto la dominazione cartaginese. I rituali documentati attraverso l'indagine stratigrafica e le analisi di laboratorio sono l'incinerazione primaria e secondaria in fossa terragna, l'inumazione in fossa e la semicombustione secondaria.

Il rituale della semicombustione è stato identificato grazie ad una serie di analisi archeometriche effettuate sui resti scheletrici rinvenuti nell'area maggiormente periferica della necropoli (fig. 4), i quali presentavano vistose tracce di annerimento localizzate sulla superficie delle ossa, pur presentandosi in buona connessione anatomica. Per tale motivo si è prontamente deciso di ricorrere all'ausilio delle analisi chimico-fisiche: le diffrattometrie ai raggi $\mathrm{X}$ e le spettrografie ai raggi infrarossi hanno precisato come la maggior parte delle salme venne sottoposta a temperature di combustione che si aggirarono attorno ai $500^{\circ} \mathrm{C}$. A fronte della totale mancanza di segni di annerimento nelle pareti delle fosse, talvolta sono stati rinvenuti minuscoli e isolati frammenti di carboncini negli strati di riempimento a più diretto contatto con le ossa: si tratta verosimilmente delle labili tracce dei carboni del rogo, introdotti all'interno della tomba contestualmente alle modalità di traslazione dei corpi, dal luogo di combustione al luogo di sepoltura. Il mantenimento delle connessioni anatomiche e lo stato di conservazione degli scheletri (fig. 5) hanno indotto a ipotizzare l'esistenza di un rituale particolare che prevedeva l'esposizione dei corpi dei defunti ad un'intensa fonte di calore per un periodo di tempo circoscritto. Il fattore-tempo è risultato dunque

Bartoloni 2010; Pompianu 2010a.

Bernardini - Zucca 2010, 41-51.

Bernardini 2000, 30-32, fig. 1:b

Attualmente la necropoli di Sirai ha restituito un numero complessivo di tombe a fossa superiore alle 325 unità; per una stima dell'estensione globale della necropoli: Guirguis 2010a, 67, nota 44. 
fondamentale per tentare di ricostruire le dinamiche del processo della semicombustione. I risultati ottenuti dai più recenti studi sugli effetti della combustione dei corpi umani (processi di alterazione dell'aspetto superficiale delle ossa e della stessa composizione anatomica del cadavere), condotti anche con l'utilizzo di moderni forni crematori ${ }^{8}$, ha consentito di ipotizzare per i defunti di Sirai un tempo di esposizione non superiore ai 30 minuti. Le analisi archeometriche, condotte col sostegno del Prof. Stefano Enzo e del Dott. Giampaolo Piga, sono state eseguite nei laboratori del Dipartimento di Chimica dell’Università degli Studi di Sassari. Grazie alla collaborazione instaurata con la Prof.ssa Assumpció Malgosa, antropologa dell'Universitat Autonoma de Barcelona, è stato successivamente avviato uno studio di dettaglio su singoli contesti della necropoli. Le metodiche di laboratorio adottate e i risultati conseguiti, sottoposti al vaglio della comunità scientifica ${ }^{9}$, rappresentano una novità nel panorama degli studi sulla ritualità fenicia e punica. In sede interpretativa, considerando un insieme eterogeneo di fattori (distribuzione nel tempo del rituale, localizzazione dei gruppi tombali, composizione e articolazione dei corredi, convergenze e divergenze rispetto alla documentazione pregressa), abbiamo ipotizzato che le deposizioni contraddistinte dall'adozione del rituale della semicombustione costituissero la testimonianza tangibile del perdurare di una "tradizione fenicia di Sardegna" ancora nella prima età punica, evolutasi per effetto dei profondi mutamenti politici e sociali conseguenti all'introduzione di nuovi gruppi di potere di estrazione cartagines $\mathrm{e}^{10}$.

Il quadro documentario ottenuto dalle ricerche condotte fino al 2007 è stato ulteriormente arricchito da altre acquisizioni. Con le indagini del triennio successivo è stata effettuata la ripulitura superficiale dei settori limitrofi dell'area segnata dalla maggior concentrazione tombale, dove affioravano dei banchi di tufo. Lo scavo stratigrafico ha mostrato il susseguirsi di strati a matrice argillosa, molto compatti, accumulatisi negli avvallamenti naturali che si interpongono tra i banchi di roccia tufacea, in parte affioranti e con escavazioni di forma sub-rettangolare dove insistono le tracce di annerimento. L'impressione iniziale ${ }^{11}$ che potesse trattarsi di un'area adibita ad ustrinum ha ricevuto conferma dalle analisi archeometriche realizzate, le quali hanno portato a concludere come le superfici annerite del tufo abbiano subito un effetto di cristallizzazione dovuto alla prolungata esposizione ad intense temperature. Durante i processi di combustione della superficie tufacea, all'interno del reticolo cristallino furono inglobati microscopici frammenti di idrossiapatite, isolati e ben riconoscibili nello spettro di diffrazione ai raggi $X$ (fig. 6); poiché, com'è noto, l'idrossiapatite $(\mathrm{HA})$ costituisce il principale componente inorganico delle ossa, gli esiti delle analisi condotte sui campioni di tufo siraiani possono considerarsi dirimenti.

La situazione documentata restituisce, dunque, un raro esempio ben conservato di un ustrinum di età fenicia e punica, utilizzato probabilmente per lungo tempo. La contiguità topografica tra questa area e le tombe con gli individui semicombusti sembra confermare le

8 Bonhert - Rost - Pollack 1998; Gómez Bellard 1996, 62.

9 Piga et al. 2010.

10 Guirguis 2010a, 189-194.

11 Guirguis 2010a, 177, figg. 355-357. 
modalità di svolgimento del processo, che probabilmente prevedeva l'uso di un sudario per il trasporto delle salme preservatesi in connessione anatomica.

Le ipotesi formulate sulle modalità di utilizzo degli ustrina si basano sulla constatazione che sono del tutto assenti a Monte Sirai, durante l'età arcaica, le incinerazioni secondarie con deposizione dei resti combusti entro urna. Le uniche tipologie tombali che prevedevano l'esposizione dei corpi all'azione del fuoco sono l'incinerazione primaria, la deposizione secondaria dei resti incinerati dentro la fossa e la semicombustione. Escludendo le incinerazioni primarie, si potrebbe pensare che l'area degli ustrina possa essere stata utilizzata anche per le incinerazioni secondarie in fossa, nonostante queste siano documentate unicamente nel settore intermedio della necropoli e soprattutto in quello meridionale, dove già da tempo furono individuate alcune lenti di bruciato non direttamente connesse ad alcuna tomba e al momento interpretabili come aree di combustione localizzate, ustrina individuali o resti di incinerazioni infantili non meglio documentabili ${ }^{12}$. Un'ulteriore ipotesi riguarda l'eventualità che gli ustrina individuati siano stati utilizzati per la cremazione degli individui infantili documentati dai numerosi enchytrismoi rinvenuti nella necropoli e relativi alla prima età punica (fig. 7). Le ultime ricerche hanno infatti dimostrato, attraverso analisi antropologiche ${ }^{13}$ e chimico-fisiche ${ }^{14}$, che la tipologia tombale dell'enchytrismos, generalmente connessa all'inumazione, almeno a Monte Sirai era utilizzata anche per la deposizione secondaria dei resti incinerati. Queste considerazioni ci inducono a collocare tra la fine del VI e il V sec. a.C. l'arco temporale di utilizzo degli ustrina individuati alla periferia nord-orientale della necropoli.

Lo stesso inquadramento cronologico di questo settore decentrato della necropoli, dove è stato individuato il numero più consistente di semicombustioni (ma anche inumazioni e deposizioni secondarie ad enchytrismos), si estende tra la fine del VI e per tutto il V sec. a.C. In prospettiva diacronica e considerando lo sviluppo topografico complessivo del sepolcreto, anche la stratificazione orizzontale delle singole sepolture dovuta alla dispersione radiale dei nuclei familiari, rende indiretta testimonianza della progressione temporale intercorsa durante l'occupazione dei diversi settori cimiteriali, dalla fine del VII fino alla metà del IV sec. a.C.

Come sostenuto di recente, i corredi della prima età punica rinvenuti a Sirai sono contraddistinti da forme ceramiche in cui prevalgono caratteristiche morfologiche di timbro conservativo, concettualmente legate alle pregresse produzioni di età arcaica, su cui si impostano, talvolta, sintassi decorative e stilistiche del tutto originali ed innovative che trovano confronti unicamente tra il repertorio del centro maggiore di Sulky ${ }^{15}$. Le datazioni proposte fino al 2007 sulla base dell'impressione generale derivante dalla presenza di indicatori cronologici interni alle produzioni vascolari di ascendenza fenicia, sono state confermate tra il 2008 e il 2009 da tre contesti tombali segnati dalla presenza di due kylikes attiche tipo C (“concave lip") e di una kylix attica a figure nere con rappresentazione del

12 Bartoloni 2000a, 83-85; Botto - Salvadei 2005, 83-84, 143.

13 Botto - Salvadei 2005, 82-83, 148-149, 164.

14 Guirguis 2010a, 164-165, 176.

15 Guirguis 2010b, 190-193, figg. 22-23. 
mito di Peleo e Teti ${ }^{16}$ (fig. 8). Tali associazioni in contesto chiuso, oltre a fornire un sicuro ancoraggio cronologico per le forme locali, dimostrano come l'orizzonte di questo settore necropolare raggiunga certamente la prima età punica.

Il rinvenimento di queste coppe d'importazione attica aiuta, inoltre, nell'interpretazione e datazione di altri contesti nei quali si rinvengono esemplari di coppe biansate con labbro distinto, da considerarsi rielaborazioni locali di forme potorie greche. L'interesse principale per tali coppe, esemplificate dalle due kylikes puniche rinvenute nelle TT. 292 e 314 (fig. 9), risiede nella constatazione che non si tratta propriamente di "imitazioni" bensì di adattamenti formali che combinano, in un medesimo esemplare, vari elementi costitutivi delle produzioni greche (breve labbro distinto, doppia ansa a maniglia) ad altri maggiormente caratteristici del repertorio fenicio e punico (piede indistinto, fondo piatto, schema decorativo). Considerata la cronologia relativa, gli esemplari che si rinvengono nelle tombe punico-arcaiche di Sirai - ma anche nei settori ipogei di Sulky ${ }^{17}$-, appaiono dunque rielaborazioni originali di coppe attiche, secondo una consuetudine diffusa e sedimentata presso le botteghe ceramiche attive su tutto il territorio sulcitano. Infatti, così come al principio del V sec. a.C. si hanno coppe biansate influenzate dalle contemporanee produzioni greche, procedendo a ritroso nel tempo abbiamo raccolto a Sirai un numero consistente di coppe analoghe relative ai corredi di alcune incinerazioni primarie del VI sec. a.C., i cui modelli di riferimento vanno ricercati nelle coeve produzioni etrusco-corinzie, in bucchero e ioniche (fig. 10). Se tutte queste produzioni ceramiche sono realmente connesse al consumo di vino, è sintomatico come tale associazione venga veicolata e supportata da un adattamento tipologico e attraversi tutta la storia degli insediamenti fenici della Sardegna, i quali accolsero forme potorie greche, rielaborandole localmente presumibilmente per gli stessi fini, fin dagli orizzonti alto-arcaici della metà dell’VIII sec. a.C.

Nell'impossibilità di ripercorrere tutti i risvolti insiti nell'intera documentazione raccolta, in questa sede si segnala una peculiarità del registro archeologico siraiano che investe una tematica di ricerca piuttosto interessante. Un dato rilevante è senza dubbio l'alta percentuale di individui deceduti in età pre-puberale. Le sepolture infantili che abbiamo scavato a Monte Sirai sono relative sia all'età arcaica che all'età punica. Sul totale delle 96 tombe individuate tra il 2005 e il 2010, i contesti riferibili a individui deceduti prima dell'età adolescenziale (entro i 12 anni) sono 26 e rappresentano, con il 27\% delle attestazioni, quasi un terzo del totale degli individui documentati. Di queste tombe solo $7 \mathrm{si}$ riferiscono ad enchytrismoi, una tipologia ben nota e molto diffusa a Sirai (fig. 7). Con uno sguardo alla documentazione pregressa relativa ad altri settori della necropoli, si nota una presenza ugualmente notevole di sepolture infantili di varia cronologia e tipologia ${ }^{18}$. Il dato è senza dubbio da integrare con l'edizione complessiva di tutti i contesti scavati, ma può comunque considerarsi di per sé piuttosto indicativo.

6 Guirguis 2007, 127-132.

7 Muscuso 2008, 15-16, fig. b:VI.

18 Bartoloni 1987; 2000a; Campanella - Martini 2000; Botto - Salvadei 2005, 143-144; Martini 2005; Guirguis 2010a. 
Tenendo presente problematiche di ampio respiro, in primo luogo quella che investe il rapporto tofet e necropoli, nonché la presenza di sepolture infantili nei cimiteri destinati agli adulti, la situazione riscontrata a Monte Sirai appare quantomeno singolare, anche a fronte della nota carenza di testimonianze di tale natura soprattutto per gli orizzonti di età arcaica. Le tombe infantili individuate mostrano, quasi costantemente, la presenza di monili, vaghi di collana molto distintivi, ma non mancano casi particolari dove si assiste alla deposizione di un corredo ceramico articolato.

Per stimolare il dibattito su un argomento molto complesso ${ }^{19}$, legato al fenomeno del cosiddetto informal burial e alla dicotomia necropoli/tofet, si sottolinea come le ultime ricerche abbiano consentito di riconoscere una marcata visibilità funeraria, espressa da un insieme di fattori che indicano con chiarezza come alcuni bambini, pur appartenenti alla sfera sociale dell'infanzia, furono seppelliti con una complessa articolazione del gesto funerario, con una "cura del sepolcro" generalmente riservata agli individui adulti, membri integranti ed effettivi della comunità di riferimento. È probabile che tale peculiarità rifletta un preciso sovrastrato culturale: la sepoltura formale, insolita per gli individui non adulti, potrebbe essere stata dettata da esigenze di tipo autorappresentativo, da un interesse interno ai gruppi familiari che sentono la necessità di preservare la visibilità funeraria anche degli individui meno rappresentativi del proprio ordinamento sociale. In altri termini la presenza elevata di tombe infantili potrebbe interpretarsi come un tentativo di rimarcare e rafforzare i legami di parentela dei nuclei familiari, rendendo pienamente visibili le linee di trasmissione di tali vincoli anche nella dimensione della ritualità funeraria.

Inoltre il confronto tra le varie caratteristiche costruttive delle tombe infantili mostra l'esistenza di casi particolari che si possono rapportare ad una condizione sociale elevata che rende ragione della complessità rituale che accompagna il gesto deposizionale (fig. 11). Operando le necessarie scansioni cronologiche, che ci testimoniano dell'evoluzione diacronica del fenomeno, si nota una certa varietà nelle soluzioni adottate per il trattamento degli individui infantili di Sirai. Nell'età arcaica sembrano prevalere, come mostrano i contesti noti delle TT. 32, 158, 163 e 251.252, le tombe bisome con i resti infantili deposti in associazione ai resti di un adulto ${ }^{20}$. In altri casi, ugualmente databili entro il VI sec. a.C., i piccoli defunti della comunità siraiana furono deposti all'interno di sepolcri ben strutturati e con corredi composti da ceramica vascolare ed elementi del corredo personale. La fragilità dei resti ossei non consente di valutare con certezza, in tutti i casi esaminati, il rituale di trattamento cui vennero sottoposte le spoglie dei bambini.

Nel dettaglio dell'evidenza raccolta durante gli ultimi sei anni di scavo le 27 tombe infantili sono rappresentate da contesti piuttosto diversificati, con cronologie che coprono pressoché tutto l'arco cronologico di riferimento della necropoli. Nei nuovi settori scavati tra il 2008 e il 2009 è stato indagato il caso particolare della T. 301 relativa all’inumazione di un soggetto deceduto in età pre-puberale (fig. 12). Nonostante il pessimo stato di conservazione dei resti scheletrici, si è potuto appurare che il corpo del defunto venne deposto in posizione laterale contratta sul fianco destro, con gli arti inferiori piegati ad

19 Sul vasto dibattito, mai sopito, si può quantomeno rimandare ad alcuni recenti lavori critici: Bernardini 2005; Xella 2010.

20 Bartoloni 1988; Botto - Salvadei 2005, 89-104, 120-123, 147; Guirguis 2010a, 25, 118-120. 
angolo retto, entro una tomba dall'inusuale apparato costruttivo. Il sepolcro è infatti realizzato con numerose lastre di calcare opportunamente sbozzate e di diverse dimensioni che formano una sorta di piccola struttura, in parte assimilabile ad una variante di cista litica. Le lastre furono accuratamente deposte sul fondo, sulle pareti della fossa e sulla copertura. Il corredo ceramico è composto da un unico pentolino al tornio miniaturizzato, mentre tra gli ornamenti personali è presente una cavigliera (rinvenuta attorno alla tibia sinistra), un orecchino di bronzo ed una collana composta da almeno 25 vaghi policromi. Il pentolino con breve ansa, orlo sottile e verticale, è caratterizzato da una bugna da presa o falso-versatoio non documentabile, peculiarità morfologica che lo accomuna ad analoghi prodotti di Monte Sirai con bugne multiple ${ }^{21}$. Questa forma ceramica conferma quanto più volte espresso in relazione alla ricorrente presenza di vasellame da cucina nelle tombe infantili e femminili dell'età arcaica ${ }^{22}$.

La tipologia tombale utilizzata per la T. 301 è di grande interesse perché documenta una nuova concezione costruttiva. Il fenomeno della giustapposizione di lastre calcaree o trachitiche lungo tutta la superficie interna delle fosse è stato riscontrato fin dal 2007 nella T. 255 e nella T. 256.258. Il rivestimento interno delle pareti delle fosse si può considerare come una variante delle tombe cosiddette "a cassone" ${ }^{23}$, mentre nel caso della T. 301 sembrerebbe trattarsi a tutti gli effetti di una cista litica.

La posizione laterale rannicchiata è una peculiarità di grande interesse che ritroviamo documentata anche per l'individuo del livello superiore della citata T. 256.258. La particolare posizione in decubito laterale contratto, sebbene piuttosto rara in Sardegna, caratterizza anche un'altra tomba infantile della necropoli siraiana (T. 50) ${ }^{24}$. Fuori dalla Sardegna si hanno testimonianze di questa pratica deposizionale a Cartagine ${ }^{25}$, a Kerkouane $^{26}$, a Ibiza ${ }^{27}$. Sebbene documentato anche in area orientale, segnatamente a Khaldé28, tale costume funebre è frequentemente attestato soprattutto nelle necropoli fenicizzate del Marocco atlantico ${ }^{29}$ e nelle necropoli del Sahel tunisino contrassegnate da dominanti pratiche funerarie «habituellement attribuées à un milieu libyque» ${ }^{30}$.

21 Si veda il pentolino frammentario della T. 42: Bartoloni 2000a: 164, fig. 34:115; gli altri esemplari sono discussi e presentati: Bartoloni 2000a, 113, figg. 32:86; 37:148; tavv. XVII:c; XXIX:b.

22 Ad esempio: Finocchi 2004, 146.

23 Guirguis 2010a, 127; per una tomba “a cassone” di età arcaica da Othoca: Del Vais 2010, 40, fig. 19.

24 Bartoloni 2000a, 72, fig. 15; Finocchi 2004, 137, nota 19.

25 Bénichou-Safar 1982, 258.

26 Nel contesto della T. 6/92 di Arg-el-Ghazouani è testimoniata la compresenza di un maschio adulto inumato in decubito laterale contratto e di una donna incinerata, considerati rispettivamente di origine libica e di origine punica o greca: M.H. Fantar 1994, 58-59; M. Fantar 2004, 118.

27 Un caso isolato e «puramente circunstancial» è segnalato nella sepoltura LIII del settore di Can Partit (Costa Ribas 1991, 48, tav. IV:4)

28 Saïdah 1966, 78, tav. IV (tomba 167).

29 Cfr. Ponsich 1967, 46-48, 55-56, 60-63, 72-78, 84-87, 92-99; figg. 12-14; 16-18; 22-26; 30-32; tav. XIII; la posizione dei defunti inumati, all'interno di fosse rivestite con lastre squadrate, è considerata estranea ai riti funerari fenici e pertanto indicativa di una popolazione autoctona secondo l'interpretazione espressa in El Azifi 1995, 404; sull'argomento cfr. anche Villada Paredes - Ramon Torres - Suárez Padilla 2010, 204; per la datazione della necropoli di Aïn Dalhia Kebira anteriormente al V sec. a.C.: Kbiri Alauoi 2000.

30 Morel 2003, 103. 
L'elevata mortalità infantile dovette dunque affliggere anche le comunità siraiane, costituendo inoltre un alto fattore di rischio per le stesse donne durante la gravidanza. A questo proposito un contesto piuttosto singolare nel panorama delle necropoli fenicie e puniche è costituito dalla T. 316 che ha accolto i resti, probabilmente inumati, di una donna gravida (fig. 13). L’individuo femminile appariva deposto con il cranio rivolto verso est. Il corredo ceramico, composto da cinque recipienti, era distribuito attorno ai resti scheletrici: una brocca con orlo espanso dietro il cranio, una brocca bilobata e una coppa sopra la mano destra, due piatti ombelicati lungo la porzione superiore dell'arto inferiore destro. Durante la rimozione dello strato terroso di riempimento, all'altezza del bacino, è stato possibile riconoscere i fragili resti ossei di un feto che, a giudicare dal solo esame autoptico e in attesa dello studio antropologico definitivo, appariva in uno stato di formazione scheletrica piuttosto avanzata. Dalla posizione riscontrata e dalle caratteristiche del riempimento, si può evincere come la decomposizione dei tessuti sia avvenuta preservando la posizione originaria del cadavere, trattandosi di una condizione tafonomica che gli specialisti definiscono “decomposizione in spazio pieno con riempimento progressivo". La situazione deposizionale riscontrata restituisce, dunque, l'immagine fedele di come dovette presentarsi il corpo della donna al momento dell'interramento. I fragili resti ossei dell'individuo non ancora nato appaiono disposti nella classica posizione fetale, almeno a giudicare dalla localizzazione dei piccoli femori rispetto alla colonna vertebrale e ai sottili frammenti di tavolato cranico. L'ipotesi che la donna possa essere deceduta per complicazioni insorte durante il processo di gravidanza, per quanto verosimile, deve essere ancora confermata dall'esame antropologico. Nel panorama delle necropoli fenicie e puniche si tratta probabilmente del primo caso di donna incinta con un'evidenza documentaria così marcata. La rarità di attestazioni di questo tipo si segnala, del resto, per differenti ambientazioni culturali e cronologiche ${ }^{31}$.

\section{L’ABITATO}

Dal 2010 le ricerche condotte nell'insediamento di Monte Sirai hanno interessato il settore meridionale dell'Insula C, ubicato in posizione decentrata rispetto all'ingresso settentrionale dell'acropoli da cui dista in linea d'aria circa $120 \mathrm{~m}$. L'indagine si è svolta su una superficie totale di circa $150 \mathrm{~m}^{2}$ composta, all'inizio dell'intervento, da circa 7 vani di differenti dimensioni (fig. 14). L'area indagata costituisce la cuspide meridionale dell'Insula abitativa centrale ed è fiancheggiata dalle due principali arterie viarie che, partendo dalla Piazza 1 del Tempio di Astarte, si immettono nella cosiddetta Piazza 3, uno spazio aperto localizzato nei pressi dell'ingresso meridionale dell'acropoli. Il settore, che abbiamo denominato C-Sud, è separato dalla Piazza 3 da un ampio muro a doppio paramento e ad andamento curvilineo che racchiude tutto il fianco sud-orientale del quartiere abitativo. Gli elementi di interesse che hanno spinto ad intraprendere le ricerche in questo settore dell'acropoli sono costituiti dalla natura/funzione del muro curvilineo, dalla vicinanza di uno spazio aperto e, infine, dalla posizione topografica decentrata rispetto alle

31 Ad esempio: Liston - Papadopoulos 2004; Malgosa et al. 2004; De Miguel Ibáñez 2008; 2010, $142-144$. 
aree d'abitato finora indagate in maniera sistematica ${ }^{32}$. Sebbene il giacimento sia stato alterato e intaccato nei livelli superficiali, a seguito di interventi di scavo e ripulitura effettuati tra la fine degli anni ' 60 e gli anni ' $80^{33}$, in tutta l'area col progresso dell'indagine si è registrata un'affidabile stratificazione. La ripulitura dei livelli superficiali e lo spietramento intensivo, attuati col fine di mettere in luce i muri perimetrali dell'abitato e le suddivisioni interne dei vari ambienti, causarono l'asporto degli ultimi strati di crollo e l'esposizione dei livelli superiori che, col passare dei decenni, costituiscono attualmente lo strato di humus danneggiato dalla vegetazione spontanea.

A causa del ridotto interramento che caratterizza tutto il pianoro, fin dalla prima campagna di scavo del 2010 si è potuta raggiungere la roccia vergine in alcuni vani ubicati nel fianco sud-occidentale di C-Sud. Il proseguimento dell'indagine con la messa in luce e lo scavo dei livelli stratigraficamente affidabili ha consentito di rilevare, nel complesso, una discontinuità d'uso dei diversi ambienti. Il piccolo vano C60S ha una cronologia di chiusura del deposito da porre latamente nella seconda metà del VI sec. a.C., mentre in C58 si è posta in luce un'importante fase di età tardo-antica e altrove si sono evidenziati, per il momento, strati di età ellenistica inquadrabili tra il IV-III e il II-I sec. a.C.

La presenza di indicatori archeologici, soprattutto ceramica vascolare, conferma la datazione della più antica presenza fenicia a Monte Sirai nella seconda metà dell'VIII sec. a.C., sebbene tali materiali provengano non da livelli cronologicamente affini, bensì dagli strati arcaici relativi alla fine del VII-metà VI sec. a.C., da quelli di età punico-ellenistica e dalle ultime fasi di media e tarda età repubblicana. Il registro archeologico subisce, al principio del I sec. a.C., una battuta d'arresto che si traduce nel totale abbandono dell'insediamento per lunghi secoli, fino alla breve ma significativa rifrequentazione in età tardo-antica (VI-VII sec. d.C.).

Come si evince dal preliminare studio dei materiali e delle sequenze stratigrafiche, l'orizzonte temporale dei primi interventi di natura antropica si inquadra non prima della metà del VII sec. a.C. ed è testimoniato da azioni di taglio, livellamento e colmata delle fenditure che caratterizzavano il banco roccioso emergente. Seppure in posizione di giacitura secondaria, la presenza relativamente abbondante di reperti ceramici la cui datazione risale almeno fino al terzo quarto dell'VIII sec. a.C. indica l'esistenza di livelli di vita alto-arcaici che potranno essere indagati col proseguimento delle ricerche. In attesa di poter precisare ulteriormente la fisionomia del primo stanziamento fenicio sul pianoro si può ipotizzare, sulla base di alcuni indizi emersi durante l'indagine stratigrafica, che i

32 Sulle indagini nel tessuto abitativo di Sirai: Guirguis - Pla Orquín in stampa; Marras 1981; Bartoloni 1994; 2000a, 53-64; Bernardini - Perra 2001; Perra 2001; Bartoloni 2002; Campanella - Finocchi 2002; Guirguis 2005, 19-22; 2010a, 63-66; sui materiali dall’abitato: Botto 1994; Peserico 1994; Balzano 1999; Campanella 1999; Perra 2000; Campanella 2002; Finocchi 2002; per i dati dal territorio: Finocchi 2005; 2007.

33 Come si evince dalla situazione stratigrafica individuata all'inizio dello scavo (restauri murari a secco, spietramento e asportazione dei livelli superficiali, tagli e colmate localizzati), gli interventi realizzati fino agli anni '80 hanno interessato alcuni punti localizzati e raramente si sono spinti in profondità; per i riferimenti noti in letteratura: Barreca 1970, 27, nota 10, fig. 2; 1984; 1986, 4; analoghe evidenze di rimaneggiamenti e restauri moderni sono state segnalate anche nel settore nord-orientale dell'Insula B: Campanella - Finocchi 2002, 47-48. 
materiali mobili di VIII-VII sec. a.C. si relazionino con le fasi di vita originariamente connesse al muro curvilineo USM 1 che delimita, verso sud, i vani C63 e C64.

I materiali di età fenicia individuati in C-Sud descrivono un arco cronologico che abbraccia tutta l'età arcaica. Tra le classi della ceramica vascolare sono attestate forme aperte in red-slip e in ceramica comune, tripodi, bacini, pentole, brocche ed anfore da trasporto. Alcuni esemplari di piatti con breve orlo arrotondato superiormente ${ }^{34}$ (fig. 15:AB) ed estroflesso ${ }^{35}$ (fig. 15:C) si riferiscono a tipologie riconducibili ai più arcaici orizzonti documentari della Sardegna fenicia che rimontano, in alcuni casi, fino alla prima metà dell'VIII sec. a.C. ma che nel contesto di Sirai pare più prudente collocare nella seconda parte dello stesso secolo. Una successiva fase che raggiunge la metà del VII sec. a.C. è documentata dall'incremento quantitativo e dalla differenziazione delle tipologie vascolari documentate, tra cui prevalgono quelle legate alla preparazione e al consumo degli alimenti, soprattutto bacini ${ }^{36}$ e tripodi ${ }^{37}$ (fig. 17:A-D), coppe carenate $^{38}$ (fig. 15:H) e a profilo emisferico ${ }^{39}$ (fig. 15:J).

Dalla seconda metà del VII sec. a.C. fino al tardo arcaismo si registra un ulteriore incremento del registro archeologico. Tra le forme aperte da mensa prevalgono i piatti ombelicati $^{40}$ (fig. 15:D-G), mentre le coppe sono documentate nelle varianti con carenatura $^{41}$ (fig. 15:M), con profilo a calotta ${ }^{42}$ (fig. 15:K) e troncoconico ${ }^{43}$ (fig. 15:L). Le anfore da trasporto sono in prevalenza riconducibili alla tipologia maggiormente caratteristica delle produzioni centro-mediterranee durante questo arco cronologico $^{44}$ (fig.

34 Botto 2009a, 99-101, nn. 2, 6-7; Pompianu 2010a, 30-31, fig. 5:7; 2010b, 1275, fig. 8:2.

35 Peserico 1994, 124-125, fig. 1:a-b; Bernardini 2000, 52, fig. 14:3; Perra 2005, 185-186, fig. 6:a; Pompianu 2010a, 29, fig. 3:1.

36 Per il bacino in fig. 6:A: Marras 1981, 197-198, fig. 6:7; Balzano 1999, 119, fig. 34:311; Bernardini 2000, 42, fig. 6:3; 2006, 144, fig. 22 (da Tratalias); Campanella 2009a, 253-254, nn. 33-36; per il bacino in fig. 6:B Balzano 1999, 116-118, fig. 33:297; Perra 2005, 188-189, fig. 9:a-b; 2007, fig. 10:8; per il bacino in fig. 6:C: Bartoloni 2000a, fig. 10, 9.

37 Bernardini 1990, 87, fig. 6:b; Campanella 2009a, 286-289, fig. 35, nn. 1-2.

38 Balzano 1999, 96, fig. 29:266.

39 Peserico 1994, 139, fig. 3:g-h; Balzano 1999, 35-37, fig. 11:132; Bernardini 2000, 43, 46, fig. 9:10-12; Secci 2006, 179, fig. 39:44; Botto 2009a, 123-124, nn. 1, 7.

40 Il piatto in fig. 15:G si può includere in una nutrita serie di forme aperte caratterizzate da effetti cromatici e disegnativi che compaiono attorno alla metà del VI e si evolvono ulteriormente fino alla prima metà del V sec. a.C., secondo una tendenza tipica dell'area sulcitana: Guirguis 2010a, 162-163, figg. 337-339; 2010b, 193, fig. 23.

41 Marras 1992, 179-180; Balzano 1999, 84-86, fig. 6:238; Bartoloni 2000a, 101, 144, fig. 25, n. 6; Finocchi 2002, 59-60, fig. 1:5.

42 Balzano 1999, 39, figg. 13:141; 14:149; Finocchi 2002, 57-58, fig. 1:1; Guirguis 2010a, 142-143, 151-152, figg. 264, 266, 291, 294.

43 Marras 1981, 192, fig. 3:1; Balzano 1999, 50, 58, fig. 19; 182-189; fig. 20; Bartoloni 2000a, 100-101, 165, fig 34, n. 116; Campanella 2000, 103-106, fig. 6; Botto - Salvadei 2005, 121-122, figg. 35:c; 41:b; per una coppa relativa ad un corredo tombale della necropoli arcaica di Sulky: Bartoloni 2009a, 73, 77, fig. 5.

44 Botto 1994; Bartoloni 2000a, 114, fig. 42:253; Guirguis 2010a, 106-108, figg. 169-160 bis; sulle analisi archeometriche delle anfore fenicie e puniche di Monte Sirai: Bordignon et al. 2005, 193-195, 203-204, 215; Botto et al. 2005, 60, 87-88 segg., tab. 2. 
15:N-P). Oltre ai bacinit ${ }^{45}$ (fig. 17:E-F) e alle pentole d'impasto e tornite, si annoverano alcune importazioni come un attacco di ansa di coppa ionica B2 ${ }^{46}$ e l'orlo di una brocca lobata in bucchero ${ }^{47}$. Tra le forme arcaiche si ha, infine, una presenza minoritaria di frammenti relativi a forme chiuse, tra cui almeno un esemplare di brocca con orlo espanso (fig. 15:S).

Tra la ceramica raccolta si segnala la presenza di alcuni coperchi (fig. 15:Q-R). L'attribuzione dei frammenti rinvenuti nello scavo del 2010 si basa sulle differenze di trattamento osservabili tra le pareti interne ed esterne, essendo le prime non ingobbiate e con le linee di tornitura in evidenza mentre le seconde appaiono lisciate a stecca, ingobbiate e verniciate; anche l'irregolare rifinitura del labbro consente di escludere altre attribuzioni. Il primo coperchio caratterizzato dal profilo a calotta (fig. 15:R), pur presente in area sulcitana come mostrano altri esemplari da Sulky e da Tratalias ${ }^{48}$, è documentato tra VII e VI sec. a.C. soprattutto a Cartagine, sia nell'abitato ${ }^{49}$ che nelle necropoli ${ }^{50}$. Il coperchio con carenatura (fig. 15:Q), anche in virtù della tonalità e consistenza della vernice riconducibile alla red-slip, potrebbe datarsi entro la metà del VII sec. a.C. La forma, piuttosto rara nel repertorio ceramico occidentale, ha qualche analogia con i coperchi di maggiori dimensioni utilizzati in associazione alle urne stamnoidi della necropoli di Bitia ${ }^{51}$, mentre dal punto di vista morfologico i migliori confronti si individuano nei piccoli coperchi utilizzati generalmente in associazione con le anfore domestiche a spalla carenata, come mostrano un esemplare di Sa Caleta a Ibiza ${ }^{52}$ e i più noti coperchi delle TT. 1 e 4 di Trayamar ${ }^{53}$. Di difficile inquadramento è la forma aperta carenata con orlo gonfio ed estroflesso (fig. 15:I) che, pur richiamandosi morfologicamente alle coppe carenate, non presenta alcun tipo di trattamento delle superfici e si caratterizza per le dimensioni notevoli. Probabilmente si tratta di una forma relativa ad una tipologia di bacini finora scarsamente documentata ed inclusa solo di recente nel repertorio ceramico sulcitano a seguito dei rinvenimenti effettuati nella T. 12, nella T. 9 PGM e nella Tomba di Via Belvedere di Sant'Antioco ${ }^{54}$.

Tra il materiale di età arcaica è senza dubbio da rimarcare la forte incidenza percentuale della ceramica d'impasto nuragica ${ }^{55}$ (fig. 17:I), sfortunatamente non sempre diagnostica. Nel vano C60S sono state individuate, in associazione con ceramica tornita, due forme d'impasto parzialmente ricomponibili (fig. 16). La prima è una pentola monoansata con

45 Per il reperto in fig. 6:E: Marras - Bartoloni - Moscati 1989, 239, fig. 5; Balzano 1999, 122-123, fig. 312; per il reperto in fig. 6:F: Bartoloni 1992; 2000a, fig. 42, n. 251.

46 Guirguis 2007, 121-127.

47 Santocchini Gerg in stampa.

48 Bernardini - D’Oriano 2001, 99-100, n. 93; Bernardini 2009, 23, 47, fig. 12:2.

49 Vegas 1999, 175, fig. 81.

50 Lancel 1982, 267-268, figg. 341-2, 399-400; Chelbi 1985, 99-100.

51 Bartoloni 1996, fig. 17:120; fig. 31:346; tav. XXIV:7.

52 Ramon 2007, 97, fig. 25:r-7.

53 Schubart - Niemeyer 1976, 212-213, tavv. 12/547:557; 16/606; Martín Ruiz 1995, 123, fig. 108; Ramon 2010, 225, 245, fig. 4:65.

54 Bernardini 2008, 565-584; Muscuso 2008, 18-19, fig. c:III; Bernardini 2010, 1265.

55 Simili ciotole e/o scodelle provengono dal nuraghe Sirai e dai livelli arcaici di Sulky: Farci 2005, 208-212, fig. 2:f; Pompianu 2010a, 32, 34, fig. 6:29. 
fondo piatto ed orlo apicato (fig. 17:H) che trova numerosi confronti nel repertorio della ceramica a mano di tipologia fenicia ma anche in quello di tradizione nuragica. In presenza di esemplari integralmente ricostruibili, l'attestazione del fondo piatto e ingrossato sembrerebbe costituire una discriminante per il riconoscimento di un apporto autoctono ${ }^{56}$. Pentole pressoché identiche sono documentate tra la ceramica da cucina dello stesso insediamento di Monte Sirai ${ }^{57}$, del nuraghe Sirai ${ }^{58}$ e di Sulky ${ }^{59}$, spesso con cronologie ancora comprese entro la seconda metà dell’VIII e la prima parte del VII sec. a.C. Il secondo esemplare rinvenuto nel vano C60S è un'anomala pentola biansata con orlo estroflesso e spalla lievemente carenata (fig. 17:G). La composizione dell'impasto, la tecnica di realizzazione e l'articolazione dell'orlo e delle anse sembrano anche in questo caso riconducibili all'ambiente nuragico del Sulcis, mentre altre caratteristiche morfologiche come la conformazione della spalla appaiono il segno di un apporto fenicio ${ }^{60}$.

Un ulteriore indizio di cultura materiale riferibile all'elemento autoctono sardo è rappresentato da una testa di mazza frammentaria, rinvenuta presso USM1 e presumibilmente reimpiegata all'interno della stessa struttura. Lo strumentario litico di questa tipologia è abbondantemente documentato in età nuragica in tutta l'isola e specialmente nella sua parte meridionale ${ }^{61}$.

Fin dalla prima campagna di scavi nel settore meridionale dell'Insula $\mathrm{C}$ sono emerse alcune tracce rapportabili ad un'attività artigianale di tipo metallurgico. Nell'angolo nordorientale del vano C60 è stato rinvenuto un forno in ceramica molto frammentario di cui residua una porzione di parete dello spessore di circa 6-7 cm e con un diametro ricostruibile superiore al metro. Considerate le dimensioni originarie e lo spessore delle pareti è plausibile ipotizzare che si tratti di un forno utilizzato per attività metallurgiche ${ }^{62}$. L'ipotesi si basa, inoltre, sulla quantità considerevole di scorie metalliche di vario peso e dimensioni, tutte apparentemente di minerale ferroso, raccolte negli strati circostanti. I frammenti di maggiori dimensioni sono stati recuperati nella soprastante unità stratigrafica di livellamento connessa all'edificazione del vano C58, il quale ha direttamente intaccato e in parte distrutto il forno. Un grosso frammento di parete, combaciante col lato meridionale superstite del tannur, risultava infatti incorporato tra le pietre dell'apparato murario di C58. Nella stessa colmata artificiale su cui si imposta il muro che delimita C58, si annovera una grande quantità di frammenti fittili relativi al forno, ridotti in frammenti amorfi di piccole dimensioni. Lo scarso materiale ceramico raccolto negli strati immediatamente al di sotto della struttura fittile, nonché i livelli connessi ad un basolato di pietre sbozzate posto in

6 Bartoloni 1985, 180; Botto 2009b, 360.

Finocchi 2002, 67, fig. 4:24

58 Perra 2007, 111-112, fig. 11:7.

59 Bartoloni 1990, 43, 70, fig. 3:121.

60 Per un'altra forma ibrida dalla T. 253 della necropoli: Guirguis - Enzo - Piga 2009, 106-109, fig. 11; Guirguis 2010b, 185-186, fig. 13

61 Usai 1988, 68, tav. IV:1-5; Bartoloni - Bondì - Marras 1992, 68-69, fig. 60; Manunza 1994, tav. V:14-16; 2002; 2008, 110, figg. 104-106, 110; una mazza di basalto frammentaria costituiva, assieme ad una scheggia di ossidiana, parte del corredo di una deposizione infantile del tofet di Sulky: Bernardini 2007, 11-12, fig. 2.

62 Lancel - Thuillier 1982, 217-260, figg. 297, 317-318, 322-327; Galeffi 2005, 989-991, figg. 1-3; Tekki 2008, 879-892, figg. 4, 6; Campanella 2009b, 470. 
opera per livellare le fenditure della roccia e rendere agibile l'area attorno al forno, sembrerebbe indicare un utilizzo di questo settore a cielo aperto fino alla seconda metà del VI sec. a.C. Ad avvalorare l'ipotesi di un'attività metallurgica contribuisce, infine, il rinvenimento di un frammento parzialmente vetrificato di tuyère $e^{63}$ recuperato in giacitura secondaria nel vano C62.

In altri settori abitativi dell'acropoli sono state rinvenute negli anni passati ingenti quantità di scorie metalliche ${ }^{64}$ che, unite alle testimonianze raccolte in C-Sud, confermano un'attività siderurgica in seno all'insediamento. Il dato è rilevante considerando il ruolo strategico svolto da Monte Sirai nel quadro di una penetrazione territoriale che si spinge fino alle porte della regione mineraria dell'Iglesiente ${ }^{65}$.

In relazione al grande muro curvilineo (USM 1) che racchiude tutto il fianco sudoccidentale dell'Insula C, la lettura planimetrica delle emergenze murarie indica che in una fase posteriore avvenne un rivestimento interno e una rettificazione dell'andamento curvilineo attraverso la parziale sovrapposizione di un ulteriore apparato murario provvisto di tramezzo centrale. In attesa di indagare i livelli connessi con tali strutture, si può segnalare la presenza di un'anfora commerciale di tipo Bartoloni D4 adagiata nell'angolo sud-orientale del vano C64. Il rinvenimento di vasellame in vernice nera di produzione attica, tra cui una kylix L. 42 A "stemless inset-lip" (fig. 18:F) databile tra il secondo quarto e la metà del V sec. a.C. ${ }^{66}$, potrebbe indiziare una cronologia da porre entro il V sec. a.C. Altre forme ceramiche riconducibili ad un orizzonte punico di V e di IV sec. a.C. sono rappresentate prevalentemente da anfore e pentole tornite con rivestimento antiaderente ${ }^{67}$ (fig. 18:A-B). Nonostante siano necessari ulteriori accertamenti stratigrafici, i dati preliminari e la lettura planimetrica del complesso consentono di collocare, indirettamente, la costruzione del muro curvilineo in un periodo anteriore al V sec. a.C.

Per quanto concerne le fasi di frequentazione posteriori si segnala l'abbondante presenza di ceramica di età ellenistica, di cui si presenta una selezione di pentole e tegami68 (fig. 18:C-D), brocche ${ }^{69}$ (fig. 18, E) e forme da mensa di imitazione attica ${ }^{70}$ (fig. 18:G-I, K-

63 Sulle varie tipologie documentate in ambito fenicio e punico: Renzi 2007, 165-178; nel Sulcis le tuyères sono documentate al nuraghe Sirai (Perra 2005, 194-195, fig. 13:b) e a Sant'Antioco, dove è stata recentemente riconosciuta un’attività siderurgica di prima metà VII sec. a.C. (Pompianu 2010b, 1273-1275, fig. 7).

64 Amadasi 1967, 56, 77; Barreca 1967, 15; Bartoloni 2000a, 62; Perra 2001, 127; Uberti 2010, 157, nota 10.

65 Bartoloni 2002; Finocchi 2005, 226; Bartoloni 2009b, 15.

66 Sparkes - Talcott 1970, 101-102, n. 469-473, fig. 5; tra le forme complete note in Sardegna si possono citare gli esemplari provenienti dai corredi della necropoli di Nora: Bartoloni - Tronchetti 1981, 109, figg. 7:41.7.1; 14:206.31.16; a Sulky in contesti d'abitato si annoverano frammenti di importazione (Unali 2010, 1230, fig. 2:a-c) e una kylix di imitazione rinvenuta nella cisterna US 500 (Campanella 2008, 163-166, n. inv. Cron 500/476); a Cagliari il tipo è assente nella necropoli di Tuvixeddu ma è documentato nei livelli abitativi di Via Brenta (Tronchetti 1993, 74, 85, tav. XXX:58-60); la maggior concentrazione di queste coppe è segnalata a Neapolis: Corrias 2005, 142, 145, tab. 4.7; dalla Sicilia punica si possono segnalare, ad esempio, i reperti di Palermo e Mozia (Di Stefano 1998, 280, 283, n. 2; Nigro 2005, 248, tav. LVII:MC.03.605/7; 2007, 232, tav. LX:MD.03.1056/55).

67 Campanella 2008, 98-101.

68 Campanella 1999, 29-33, fig. 1:2-6; Finocchi 2002, 68-70, fig. 5:26.

69 Finocchi 2002, 63-64, fig. 3:17.

70 Finocchi 2002, 63, fig. 3:13-14. 
L). I dati raccolti sul terreno indicano che l'abbandono dell'insediamento punico-romano avvenne entro i primi decenni del I sec. a.C., come sembrerebbero suggerire i pochi indicatori ceramici di tale cronologia, nello specifico un orlo di coppetta in pasta grigia ${ }^{71}$, isolati frammenti non diagnostici di sigillata aretina e un orlo di anfora da trasporto italica inquadrabile tra i tipi Dressel IB-IC ${ }^{72}$ (fig. 18:J).

Dopo un lungo periodo di abbandono si registra, come sottolineato sopra, un'estesa opera di livellamento delle emergenze murarie, funzionale all'edificazione del vano C58, con potenti colmate di terra prelevata dall'area circostante il vano C60. Tali strati contenevano soprattutto materiali riconducibili alle fasi fenicie e puniche nonché numerose scorie ferrose. Il termine ultimo per la formazione del deposito è tuttavia stabilito da alcuni reperti attribuibili latamente al VI-VII sec. d.C. tra cui si segnala un elemento di cerniera in bronzo e numerosi frammenti di forme chiuse decorate con striature parallele ad andamento curvilineo tipiche del periodo. Considerando l'articolazione complessiva di C-Sud si nota come i muri perimetrali del vano C58, caratterizzati da un andamento curvilineo e irregolare, appaiano architettonicamente autonomi rispetto alle emergenze murarie più antiche solo in parte sfruttate per la nuova progettazione, che infatti insiste ad una quota sensibilmente più alta. L'identificazione di questo edificio al momento isolato conferma la frequentazione, discontinua, del sito di Monte Sirai in età tardo-antica, come noto da tempo sulla base di alcuni reperti sporadici ${ }^{73}$ e ora documentato anche a livello stratigrafico ed edilizio.

\section{CONSIDERAZIONI CONCLUSIVE}

L'articolazione complessiva delle emergenze archeologiche che insistono sul pianoro e la continuità delle indagini, che vi si sono svolte nell'arco di circa mezzo secolo, restituiscono un quadro sufficientemente chiaro della storia del centro fenicio e punico di Monte Sirai, attivo fin dalla seconda metà dell'VIII e abbandonato tra la fine del II e gli inizi del I sec. a.C. Rimangono tuttavia ancora irrisolte alcune problematiche che concernono aspetti di dettaglio sui quali occorrerà lavorare ancora. Dalle indagini nel tofet e da quelle, ancora in corso di svolgimento, nelle necropoli e nell'abitato sono sorte e continueranno ad emergere le tessere documentarie che andranno opportunamente inserite a comporre quel mosaico di relazioni flessibili e articolate che caratterizzò la vita dei principali insediamenti della Sardegna sud-occidentale tra la fine dell'Età del Bronzo e l’Età Romana.

Dagli albori dell'età arcaica, di cui sono eccellente manifestazione le più profonde stratigrafie di Sulky e le tombe di Portoscuso, la presenza fenicia nella Sardegna sudoccidentale assume una fisionomia peculiare che si manifesta nella creazione di nuovi centri insediativi ma anche nella frequentazione - con modi, tempi ed esiti tutt'altro che definitivamente chiariti - di quei centri nuragici gestiti da un’élite sarda capace di imporsi

71 Classe ceramica diffusa a partire dalla fine del II sec. a.C. (Tronchetti 1988; Tronchetti 1996, 32-33); produzioni analoghe sono state rinvenute nel territorio attorno a Monte Sirai (Bartoloni 2000b, 15, figg. 5-6); dall'abitato: Barreca 1964, 57; Amadasi 1966, 85-86

72 Pianu 1980, 17-18; Sechi 2006, 171-174; Spanu 2006, 97, 128; Franceschi 2009, 735-736, fig. 3:12.

73 Bartoloni 2000a, 45. 
come controparte delle transazioni commerciali e come interlocutrice con cui intrattenere una profonda dialettica di natura insieme politica e culturale.

Lo studio delle trasformazioni subite dagli assetti territoriali in prospettiva diacronica rappresenta uno snodo cruciale per intraprendere un percorso conoscitivo che conduca ad una ricomposizione coerente e organica di quella complessità di fondo che avvertiamo a livello archeologico. Concentrando l'attenzione sul distretto territoriale ruotante attorno alla città di Sulky, si percepisce come la prima strutturata presenza fenicia condusse in breve tempo alla creazione di un alto numero di insediamenti che la ricerca archeologica ha fatto emergere solo in parte. Intuiamo quanto forte dovette essere stato il rapporto di fusione culturale ed etnica tra la componente di derivazione orientale e quella autoctona, come si evince dal dinamismo di alcuni centri nuragici ancora attivi fino alle soglie dell'età punica $\mathrm{e}$ dalla nascita di nuovi nuclei abitativi di timbro levantino.

Cercando di ricostruire in maniera organica la collocazione di tali punti focali della presenza umana nel territorio e nell'ottica di sondare un'eventuale differenziazione alla base della pluralità di scelte insediative, emerge un paesaggio complesso del quale si cominciano a riconoscere gli elementi di base. Pur ipotizzando una preesistente strutturazione di età nuragica in quei siti dove sorgeranno dall'VIII sec. a.C. i centri fenici, si può sottolineare come questi ultimi insistano su posizioni strategiche legate essenzialmente al controllo delle vie di transito, a fronte di una presenza maggiormente radicata e insieme parcellizzata nel territorio che è caratteristica peculiare dei centri di tradizione nuragica, essi stessi "rivitalizzati" sotto l'impulso dei contatti venutisi a creare tra le due etnie. In altre parole l'elezione di punti di riferimento stabili con la creazione di centri di popolamento sembra incidere profondamente sul tessuto connettivo della regione, tanto da far pensare ad un vero e proprio «modello di popolamento regionale» ${ }^{74}$. I principali poli della presenza fenicia, in maniera non casuale situati lungo i punti di transito, controllavano le grandi vie del commercio terrestre (Monte Sirai, Pani Loriga) e marittimo (Sulky, Inosim), secondo una strategia di gestione del territorio che è difficile concepire senza il presupposto di un intenzionale e regolato piano di gestione di tutte le risorse disponibili, agropastorali e soprattutto minerarie. Un siffatto schema organizzativo coinvolse senza dubbio i gruppi egemoni sardi detentori delle stesse chiavi di accesso a tali risorse, come risulta dalle ricostruzioni storiche più accreditate che individuano nell'elemento nuragico il principale protagonista dello sfruttamento e della raccolta delle materie prime, delegando alla componente fenicia la responsabilità della commercializzazione anche trans-marittima ${ }^{75}$. Non stupisce, dunque, il moltiplicarsi delle testimonianze di tradizione nuragica nei centri principali dell'irradiazione levantina, né la parallela presenza di cultura materiale fenicia in quei centri sardi che giocarono un ruolo non marginale nel nuovo assetto territoriale della Sardegna sud-occidentale fino alla fine del VI sec. a.C., quando le mutate condizioni politiche conseguenti all'egemonia cartaginese produssero un sensibile riequilibrio dei processi gestionali in favore di un potere centralista che appare, almeno in una prima fase e limitatamente al Sulcis, privilegiare i grandi centri a discapito del popolamento rurale. Tale è il dato che si può

74 Finocchi 2005, 254.

75 Bartoloni 2002, 44-45; Botto 2004-2005, 19; Bartoloni 2009b, 12. 
desumere dagli studi sul territorio effettuati da Stefano Finocchi che registra un totale spopolamento delle campagne di Monte Sirai a partire dalla prima età punica ${ }^{76}$, con una ripresa dell'interesse cartaginese per le aree rurali non anteriore alla metà del IV sec. a.C. in leggero ritardo rispetto ad altre regioni della Sardegna centro-meridionale dove si registra una diversificazione produttiva fin dal tardo V-inizi IV sec. a.C. ${ }^{77}$.

Le più recenti ricerche a Monte Sirai hanno consentito di ottenere sul fronte delle necropoli nuovi e significativi dati sull'articolazione delle diverse pratiche funerarie attestate per circa tre secoli, dalla fine del VII alla metà del IV sec. a.C. I risultati ottenuti, oltre a restituire un nucleo consistente ed eterogeneo di cultura materiale, incrementano in maniera sensibile i dati indispensabili per affrontare numerose problematiche che ancora attendono risposte esaustive dalla ricerca archeologica: tematiche legate al rapporto tra incinerazione e inumazione, alla presenza/assenza di deposizioni infantili nelle necropoli degli adulti, alle dinamiche sociali, alle tipologie tombali e, infine, alle espressioni della gestualità rituale.

La ripresa delle indagini nella parte meridionale dell'abitato, sebbene ad uno stadio iniziale, getta nuova luce sui secoli di frequentazione non documentati nella necropoli, vale a dire sulle prime fasi posteriori alla fondazione dell'insediamento e sugli ultimi livelli di vita di età punico-ellenistica e repubblicana. Per quanto riguarda la fase di passaggio tra l'arcaismo e la prima età punica si possiedono al momento solo alcuni indizi di natura stratigrafica e reperti mobili di V sec. a.C., di contro alla consistente documentazione restituita dai settori cimiteriali. Infine, le testimonianze di attività metallurgica riscontrate nel settore C-Sud rappresentano delle acquisizioni di grande interesse che qualificano ulteriormente la presenza fenicia e punica sul pianoro di Monte Sirai.

\section{BIBLIOGRAFIA}

AMADASI, M. G.

1966 L'abitato: M.G. AMADASI - F. BARRECA ET ALII, Monte Sirai - III. Rapporto preliminare della Missione archeologica dell'Università di Roma e della Soprintendenza alle Antichità di Cagliari (Studi Semitici 20), Roma 1966, pp. 83-104.

1967 La zona C: M.G. AMADASI - F. BARRECA ET ALII (a cura di), Monte Sirai - IV. Rapporto preliminare della Missione archeologica dell'Università di Roma e della Soprintendenza alle Antichità di Cagliari (Studi Semitici 25), Roma 1967, pp. 55-94.

BALZANO, G.

1999 Ceramica fenicia di Monte Sirai. Le forme aperte del vano C 33 (Rivista di studi fenici Supplemento 27), Roma 1999.

Finocchi 2005, 254-255.

77 Sulle recenti indagini nel sito di Truncu’ e Molas (Terralba): Van Dommelen - Gómez Bellard - Pérez Jordà 2010; Pérez Jordà et al. 2010. 
BARRECA, F.

1964 Gli scavi: F. BARRECA - G. GARBInI, Monte Sirai - I. Rapporto preliminare della Missione archeologica dell'Università di Roma e della Soprintendenza alle Antichità di Cagliari (Studi Semitici 11), Roma 1964, pp. 11-64.

1967 Il Mastio: M.G. AMADASI - F. BARRECA ET ALII (a cura di), Monte Sirai - IV. Rapporto preliminare della Missione archeologica dell'Università di Roma e della Soprintendenza alle Antichità di Cagliari (Studi Semitici 25), Roma 1967, pp. 7-26.

1970 Ricerche puniche in Sardegna: AA. VV., Ricerche puniche nel Mediterraneo centrale, Relazioni del colloquio in Roma, 5-7 maggio 1969 (Studi Semitici 36), Roma 1970, pp. 21-38.

1984 Venti anni di scavi a Monte Sirai: Nuovo Bullettino Archeologico Sardo 1 (1984), pp. 143-157.

1986 L'attività della Soprintendenza archeologica per le province di Cagliari e Oristano (19701986): Quaderni della Soprintendenza Archeologica per le Province di Cagliari e Oristano 2 (1986), pp. 3-18.

BARTOLONI, P

1985 Nuove testimonianze arcaiche da Sulcis: Nuovo Bullettino Archeologico Sardo 2 (1985), pp. 167-192.

1987 La tomba 54 della necropoli arcaica di Monte Sirai: Quaderni della Soprintendenza Archeologica per le Province di Cagliari e Oristano 4 (1987), pp. 153-160.

1988 Tracce di coltura della vite nella Sardegna fenicia: AA. VV., Stato, economia e lavoro nel Vicino Oriente antico, Milano 1988, pp. 410-413.

1990 S. Antioco: area del Cronicario (campagne di scavo 1983-86). I recipienti di uso domestico e commerciale: Rivista di studi fenici 18 (1990), pp. 37-80.

1992 Nora I. Nota su due frammenti di bacino di tipo fenicio-cipriota: Quaderni della Soprintendenza Archeologica per le Province di Cagliari e Oristano 9 (1992), pp. 99-103.

1994 Monte Sirai 1. Gli scavi del 1990-92: Rivista di studi fenici 22 (1994), pp. 75-82.

1996 La necropoli di Bitia - I (Collezione di studi fenici 38), Roma 1996.

2000a La necropoli di Monte Sirai - I (Collezione di Studi Fenici 41), Roma 2000.

2000b Il santuario di Su Campu 'e Sa Domu: P. Negri SCAFA - P. GENTiLi (a cura di), Donum Natalicium. Studi presentati a Claudio Saporetti in occasione del suo $60^{\circ}$ compleanno, Roma 2000, pp. 13-22.

2002 Monte Sirai 1999-2000. Nuove indagini nell'Insula B: Rivista di studi fenici 30 (2002), pp. 41-46.

2009a Testimonianze dalla necropoli fenicia di Sulky: Sardinia, Corsica et Baleares Antiquae 7 (2009), pp. 71-80.

2009b Miniere e metalli nella Sardegna fenicia e punica: Sardinia, Corsica et Baleares Antiquae 7 (2009), pp. 11-18.

2010 Nuove cronologie sulcitane: A. FERJAOUI (ed.), Carthage et les autochtones de son empire du temps de Zama, Hommage à Mhamed Hassine Fantar, Tunis 2010, pp. 37-40.

BARTOLONI, P. - BONDì, S.F. - MARRAS, L.A.

1992 Monte Sirai (Itinerari - IX; Istituto Poligrafico e Zecca dello Stato), Roma 1992.

BARTOLONI, P. - TRONCHETTI, C.

1981 La necropoli di Nora (Collezione di studi fenici 12), Roma 1981.

BÉNICHOU-SAFAR, H.

1982 Les tombes puniques de Carthage. Topographie, structures, inscriptions et rites funéraires, Paris 1982. 
BERNARDINI, P.

1990 S. Antioco: area del Cronicario (campagne di scavo 1983-86). La ceramica fenicia: forme aperte: Rivista di studi fenici 18 (1990), pp. 81-98.

2000 I Fenici nel Sulcis. La necropoli di San Giorgio di Portoscuso e l'insediamento del Cronicario di Sant'Antioco: P. BARTOLONI - L. CAMPANElla (a cura di), La ceramica fenicia di Sardegna. Dati, problematiche, confronti. Atti del Primo Congresso Internazionale Sulcitano, Sant'Antioco 19-21 settembre 1997 (Collezione di studi fenici 40), Roma 2000, pp. 29-62.

2005 Per una rilettura del santuario tofet - I: il caso di Mozia: Sardinia, Corsica et Baleares Antiquae 3 (2005), pp. 55-70.

2006 La regione del Sulcis in età fenicia: Sardinia, Corsica et Baleares Antiquae 4 (2006), pp. 109-150.

2007 Nuragici, Sardi e Fenici tra storia (antica) e ideologia (moderna): Sardinia, Corsica et Baleares Antiquae 5 (2007), pp. 11-30.

2008 La morte consacrata. Spazi, rituali e ideologia nella necropoli e nel tofet di Sulky fenicia e punica: X. Dupré RAVentos - S. Ribichini - S. Verger (a cura di), Saturnia Tellus. Definizioni dello spazio consacrato in ambiente etrusco, italico, fenicio-punico, iberico e celtico. Atti del Congresso Internazionale, Roma 10-12 novembre 2004, Roma 2008, pp. 565-584.

2009 Dati di cronologia sulla presenza fenicia e punica in Sardegna (IX-V sec. a.C.): Sardinia, Corsica et Baleares Antiquae 7 (2009), pp. 19-70.

2010 Aspetti dell'artigianato funerario punico di Sulky. Nuove evidenze: M. Milanese - P. RUGGERI - C. VISMARA (a cura di), L'Africa romana. I luoghi e le forme dei mestieri e della produzione nelle province africane. Atti del XVIII convegno di studio, Olbia, 11-14 dicembre 2008 (Pubblicazioni del Centro di Studi Interdisciplinari sulle province romane dell’Università degli Studi di Sassari 36), Roma 2010, pp. 1257-1266.

BERNARDINI, P. - D'ORIANO, R.

2001 Argyróphleps nesos. L'isola dalle vene d'argento, Fiorano Modenese 2001.

BERNARDini, P. - PERRA, C.

$2001 \quad$ Monte Sirai. Le opere e i giorni, Carbonia 2001.

BERNARDINI P. - ZUCCA, R.

2010 Indigeni e Fenici nelle isole di San Vittorio e Mal di Ventre (Sardegna occidentale): A FERJAOUI (ed.), Carthage et les autochtones de son empire du temps de Zama, Hommage à Mhamed Hassine Fantar, Tunis 2010, pp. 41-54.

BONHERT, M. - Rost, T. - POLlack, S.

1998 The Degree of Destruction of Human Bodies in Relation to the Duration of the Fire: Forensic Science International 95 (1998), pp. 11-21.

BORDIGNON, F. ET ALII

2005 Identificazione e studio di residui organici su campioni di anfore fenicie e puniche provenienti dalla Sardegna sud-occidentale: Mediterranea 2 (2005), pp. 189-218.

Bотто, $\mathrm{M}$.

1994 Monte Sirai 1. Analisi del materiale anforico relativo alle campagne di scavo 1990 e 1991: Rivista di studi fenici 22 (1994), pp. 83-116.

2004-2005 Da Sulky a Huelva: considerazioni sui commerci fenici nel Mediterraneo antico: Annali di Archeologia e Storia Antica 11-12 (2004-2005), pp. 9-27.

2009a La ceramica da mensa e da dispensa fenicia e punica: J. BonEtTo - G. FALEzzA - A.R. GHiotTo (a cura di), Nora. Il Foro romano. Storia di un'area urbana dall'età fenicia alla tarda antichità (1997-2006), II. 1. I materiali preromani (Scavi di Nora, I), Padova 2009, pp. 97-237. 
2009b La ceramica fatta a mano: J. BonetTo - G. FALEZZA - A.R. GhiotTo (a cura di), Nora. Il Foro romano. Storia di un'area urbana dall'età fenicia alla tarda antichità (1997-2006), II. 1. I materiali preromani (Scavi di Nora, I), Padova 2009, pp. 359-372.

BoTTO, M. ET ALII

2005 Caratterizzazione di anfore fenicie e puniche mediante analisi archeometriche: Mediterranea 2 (2005), pp. 57-110.

BotTo, M. - SALVADEI, L.

2005 Indagini alla necropoli arcaica di Monte Sirai. Relazione preliminare sulla campagna di scavi del 2002: Rivista di studi fenici 33 (2005), pp. 81-167.

CAMPANELla, L.

1999 Ceramica punica di età ellenistica da Monte Sirai (Collezione di studi fenici 39), Roma 1999.

2000 Necropoli fenicia di Monte Sirai. Il contesto della tomba 90: P. BARTOLONI - L. Campanella (a cura di), La ceramica fenicia di Sardegna. Dati, problematiche, confronti. Atti del Primo Congresso Internazionale Sulcitano, Sant'Antioco 19-21 settembre 1997 (Collezione di studi fenici 40), Roma 2000, pp. 99-107.

2002 I «reperti speciali»: Rivista di studi fenici 30 (2002), pp. 79-106.

2008 Il cibo nel mondo fenicio e punico d'Occidente. Un'indagine sulle abitudini alimentari attraverso l'analisi di un deposito urbano di Sulky in Sardegna (Collezione di studi fenici 43), Roma 2008.

2009a La ceramica da preparazione fenicia e punica: J. BonetTO - G. FALEZZA - A.R. GHIOTTO (a cura di), Nora. Il Foro romano. Storia di un'area urbana dall'età fenicia alla tarda antichità (1997-2006), II. 1. I materiali preromani (Scavi di Nora, I), Padova 2009, pp. 247-294.

2009b I forni, i fornelli e i bracieri fenici e punici: J. BonETTO - G. FALEZzA - A.R. GHIOTTO (a cura di), Nora. Il Foro romano. Storia di un'area urbana dall'età fenicia alla tarda antichità (1997-2006), II. 1. I materiali preromani (Scavi di Nora, I), Padova 2009, pp. 469-498.

CAMPANELLA, L. - FinOCCHI S.

2002 Monte Sirai 1999-2000. L’indagine stratigrafica: Rivista di studi fenici 30 (2002), pp. 4756.

CAMPANELla, L. - MARTini, D.

2000 Monte Sirai: una sepoltura infantile: Rivista di studi fenici 28 (2000), pp. 35-56.

CHELBI, F.

1985 Carthage. Découverte d’un tombeau archaïque à Junon: Revue des Études PhéniciennesCORRIAS, F. Puniques et des Antiquités libyques 1 (1985), pp. 95-117.

2005 La ceramica attica in Sardegna: R. ZuCCA (a cura di), Splendidissima Civitas Neapolitanorum, Roma 2005, pp. 135-158.

Costa RiBAs, B.

1991 Las excavaciones arqueólogicas en el solar n. 38 de la Vía Romana (Can Partit). Nuevos datos para el conocimiento de la necrópolis del Puig des Molins: AA. VV., I-IV Jornadas de Arqueología fenicio-púnica - Ibiza 1986-1989 (Trabajos del Museo Arqueológico de DEL VAIS, C. Ibiza, 24), Ibiza 1991, pp. 29-58.

2010 L'abitato fenicio-punico e romano: R. CORONEo (a cura di), La Cattedrale di Santa Giusta. Architettura e arredi dall'XI al XIX secolo, Cagliari 2010, pp. 35-46. 
De Miguel IBÁÑEZ, M.P.

2008 Gestantes en contextos funerarios altomedievales navarros: Lucentum 27 (2008), pp. 233242.

2010 Una visión de la infancia desde la osteoarqueología: de la Prehistoria reciente a la Edad Di STEFANO, C.A. Media: Complutum 21 (2010), pp. 135-154.

1998 Ceramica a vernice nera: AA.VV., Palermo punica, Palermo 1998, pp. 280-293.

EL AZIFI, M.R.

1995 Les nécropoles de la région de Tanger sont-elles phéniciennes?: M.H. FANTAR - M. GHAKI (a cura di), Actes du III Congrès International des Études Phéniciennes et Puniques, Tunis 11-16 novembre 1991, Tunis 1995, pp. 401-414.

FANTAR, M.

2004 La présence grecque dans le paysage funéraire de Carthage: Revue des Études FANTAR, M.H Phéniciennes-Puniques et des Antiquités libyques 13 (2004), pp. 113-118.

1994 Fouilles à Kerkouane: Bullettin de Correspondance et des Traveaux Hellenique-Afrique du Nord 23 (1994), pp. 51-60.

FARCI, F.

2005 Nuraghe Sirai di Carbonia. Materiali ceramici di produzione indigena dalla US 62: Rivista di studi fenici 33 (2005), pp. 207-216.

FINOCCHI, S.

2002 Monte Sirai 1999-2000. I materiali ceramici: Rivista di studi fenici 30 (2002), pp. 57-78.

2004 La necropoli fenicia di Monte Sirai: alcune osservazioni sulla distribuzione spaziale del sepolcreto e sulla visibilità "funeraria” dei defunti: Daidalos 6 (2004), pp. 133-146.

2005 Ricognizione nel territorio di Monte Sirai: Rivista di studi fenici 33 (2005), pp. 225-260.

2007 Alcuni dati sullo sfruttamento agricolo del territorio di Monte Sirai: Daidalos 8 (2007), pp. $49-60$.

FRANCESCHI, E.

2009 Le anfore romane: J. BonetTo - G. FalezzA - A.R. GhiotTo (a cura di), Nora. Il Foro romano. Storia di un'area urbana dall'età fenicia alla tarda antichità 1997-2006, Vol. GALEFFI, C. II.2 - I materiali romani e gli altri reperti (Scavi di Nora I), Padova 2009, pp. 733-746.

2005 Nuove ricerche sulle fornaci di Tharros: A. SpANÒ Giammellaro (a cura di), Atti del V Congresso Internazionale di Studi Fenici e Punici, Marsala-Palermo, 2-8 ottobre 2000, Palermo 2005, pp. 989-991.

GómEZ BELLARD, F.

1996 El análisis antropológico de las cremaciones: M. ${ }^{a}$ ANGELES QuEROL - T. CHAPA (edd.), Homenaje al Profesor Manuel Fernánedz-Miranda, vol. II (Saguntum Extra 6), Valencia GUIRGUIS, M. 1996, pp. 55-64.

2005 Storia degli studi e degli scavi a Sulky e a Monte Sirai: Rivista di studi fenici 33 (2005), pp. 13-30.

2007 Contesti funerari con ceramica ionica e attica da Monte Sirai (campagne di scavo 20052008): Sardinia, Corsica et Baleares Antiquae 5 (2007), pp. 121-132.

2010a Necropoli fenicia e punica di Monte Sirai. Indagini archeologiche 2005-2007 (Studi di Storia Antica e Archeologia 7), Ortacesus 2010.

2010b Il repertorio ceramico fenicio della Sardegna: differenziazioni regionali e specificità evolutive: L. Nigro (a cura di), Motya and the Phoenician Ceramic Repertoire between the Levant and the West $9^{\text {th }}-6^{\text {th }}$ century BC. Proceedings of the International Conference 
held in Rome, $26^{\text {th }}$ February 2010 (Quaderni di Archeologia Fenicio-Punica V), Roma 2010, pp. 173-210.

Guirguis, M. - EnZO, S. - PigA, G.

2009 Scarabei dalla necropoli fenicia e punica di Monte Sirai. Studio crono-tipologico e archeometrico dei reperti rinvenuti tra il 2005 e il 2007: Sardinia, Corsica et Baleares Antiquae 7 (2009), pp. 101-116.

Guirguis, M. - Pla ORQuín, R.

in stampa Notizie preliminari sullo scavo del 2010 nell'acropoli di Monte Sirai: M.B. Cocco - A. GAVINI (a cura di), L’Africa romana XIX, Sassari 14-17 dicembre 2010, in stampa.

KBIRI AlAOUI, M.

2000 À propos de la chronologie de la nécropole rurale d'Aïn Dalia Lekbira (région de Tanger, Maroc): M.E. Aubet - M. BARTHelemy (a cura di), Actas del IV Congreso Internacional de Estudios Fenicios y Púnicos, Cádiz 2 al 6 de Octubre de 1995, Cádiz 2000, pp. 11851196.

LANCEL, S.

1982 Les niveaux funéraires: S. LANCEL (ed.), Byrsa II. Rapports préliminaires sur les fouilles 1977-1978: niveaux et vestiges puniques (Collection de l'École Française de Rome 41), Roma 1982, pp. 263-364.

LANCEL, S. - THUILLIER, J.-P.

1982 Les niveaux d'ateliers métallurgiques: S. LANCEL (ed.), Byrsa II. Rapports préliminaires sur les fouilles 1977-1978: niveaux et vestiges puniques (Collection de l'École Française de Rome 41), Roma 1982, pp. 170-212.

LiSTON, M.A. - PAPADOPOULOS, J.K.

2004 The "rich athenian lady" was pregnant. The Anthropology of a Geometric Tomb Reconsidered: Hesperia 73 (2004), pp. 7-38.

MaLgosa, A. ET ALII

2004 A Dystocic Childbirth in the Spanish Bronze Age: International Journal of MANUNZA, M.R. Osteoarchaeology 14 (2004), pp. 98-103.

1994 Sito nuragico a Rio Paiolu. Settimo San Pietro (CA): Quaderni della Soprintendenza Archeologica per le Province di Cagliari e Oristano 11 (1994), pp. 73-88.

2002 Testimonianze di cultura materiale dal Bronzo medio all'età del Ferro nell'agro di Sinnai: Quaderni della Soprintendenza Archeologica per le Province di Cagliari e Oristano 19 (2002), pp. 17-102.

2008 La presenza dell'uomo nella preistoria: C. PERRA (a cura di), Museo Archeologico "Villa Sulcis” Carbonia. Guida alle esposizioni, Carbonia 2008, pp. 82-111.

MARRAS, L.A.

1981 Saggio di esplorazione stratigrafica nell'acropoli di Monte Sirai: Rivista di studi fenici 9 (1981), pp. 187-209.

1992 Nota su una tazza carenata dalla necropoli ad incinerazione di Monte Sirai: Rivista di studi fenici 20 (1992), pp. 179-180.

MARRAs, L.A. - BARTOLONI, P. - MOSCATI, S.

1989 Cuccureddus: Rendiconti dell’Accademia Nazionale dei Lincei 42, Roma 1989, pp. 225248.

MARTÍN RUIZ, J.A.

1995 Catálogo documental de los fenicio en Andalucía, Sevilla 1995. 
MARTINI, D.

2005 Gioielli dalla necropoli di Monte Sirai: A. Spanò GiammelLARo (a cura di), Atti del V Congresso Internazionale di Studi Fenici e Punici, Marsala-Palermo, 2-8 ottobre 2000, Palermo 2005, pp. 1071-1080.

MOREL, J.-P.

2003 De quelques survivances protohistoriques dans la Carthage punique: M. KHANOussi (ed.), Actes du VIII Colloque International sur l'Histoire et l'Archaéologie de l'Afrique du Nord (Tabarka, 8-13 Mai 2000), Tunis 2003, pp. 99-114.

Muscuso, S.

2008 Il Museo "Ferruccio Barreca” di Sant'Antioco: le tipologie vascolari della necropoli punica: Sardinia, Corsica et Baleares Antiquae 6 (2008), pp. 9-40.

NigRO, L.

2005 Zona C. Il Kothon. Catalogo e Tavole: L. Nigro (a cura di), Mozia - XI. Il Tempio del Kothon. Rapporto preliminare delle campagne di scavi XXIII e XXIV (2003-2004) condotte congiuntamente con il Servizio Beni Archeologici della Soprintendenza Regionale per i Beni Culturali e Ambientali di Trapani (Quaderni di Archeologia FenicioPunica II), Roma 2005, pp. 135-502.

2007 Zona D. Catalogo e Tavole: L. Nigro (a cura di), Mozia - XII. Zona D. La "Casa del sacello domestico", il "Basamento meridionale" e il Sondaggio stratigrafico I. Rapporto preliminare delle campagne di scavi XXIII e XXIV (2003-2004) condotte congiuntamente con il Servizio Beni Archeologici della Soprintendenza Regionale per i Beni Culturali e Ambientali di Trapani (Quaderni di Archeologia Fenicio-Punica III), Roma 2007, pp. 111-304.

PÉREZ JORDÀ, G. ET ALII

2010 La alimentación en una granja púnica de Cerdeña: C. MATA PARREÑo - G. PÉREz JoRdÀ J. ViVES-FERRÁNDIZ SÁNCHEZ (edd.), De la cuina a la taula. IV Reunió d'Economia en el Primer Millenni aC (Saguntum, extra-9), Valencia 2010, pp. 295-302.

PERRA, C.

2000 Brocche bilobate arcaiche dall'abitato di Monte Sirai: P. BARTOLONI - L. CAMPANELLA (a cura di), La ceramica fenicia di Sardegna. Dati, problematiche, confronti. Atti del Primo Congresso Internazionale Sulcitano, Sant'Antioco 19-21 settembre 1997 (Collezione di studi fenici 40), Roma 2000, pp. 259-268.

2001 Monte Sirai. Gli scavi nell'abitato 1996-1998: Rivista di studi fenici 29 (2001) pp. 121130.

2005 Una fortezza fenicia presso il Nuraghe Sirai di Carbonia. Gli scavi 1999-2004: Rivista di studi fenici 33 (2005), pp. 169-206.

2007 Fenici e Sardi nella fortezza del nuraghe Sirai di Carbonia: Sardinia, Corsica et Baleares Antiquae 5 (2007), pp. 103-120.

Peserico, A.

1994 Monte Sirai 1. La ceramica fenicia: le forme aperte: Rivista di studi fenici 22 (1994), pp. 117-144.

PiANU, G.

1980 Contributi ad un corpus anforario della Sardegna. Le anfore rodie e le anfore Dressel I e PIGA, G. ET ALII Dressel 2/4: Archivio Storico Sardo 31 (1980), pp. 11-28.

2010 A Funerary Rite Study in the Phoenician-Punic Necropolis of Mount Sirai (CarboniaSardinia-Italy): International Journal of Osteoarchaeology 20 (2010), pp. 144-157. 
POMPIANU, E.

2010a I Fenici a Sulky: nuovi dati dal vano IIE dell'area del Cronicario: Sardinia, Corsica et Baleares Antiquae 8 (2010), pp. 27-36.

2010b Un impianto artigianale per la lavorazione del ferro dall'antica Sulky (Sardegna): M. MiLANESE - P. RUGGERI - C. VISMARA (a cura di), L'Africa romana. I luoghi e le forme dei mestieri e della produzione nelle province africane. Atti del XVIII convegno di studio,Olbia, 11-14 dicembre 2008 (Pubblicazioni del Centro di Studi Interdisciplinari sulle province romane dell’Università degli Studi di Sassari 36), Roma 2010, pp. 12671282.

PONSICH, M.

1967 Nécropoles phéniciennes de la region de Tanger (Études et travaux d'archéologie marocaine 3), Tanger 1967.

RAMON, J.

2007 Excavaciones arqueológicas en el asentamiento fenicio de Sa Caleta (Ibiza) (Cuadernos de Arqueología Mediterránea 16), Barcelona 2007.

2010 La cerámica fenicia del Mediterráneo extremo-occidental y del Atlántico (s. VIII $-1^{\mathrm{r}} 1 / 3$ del VI aC). Problemas y perspectivas actuales: L. NigRo (a cura di), Motya and the Phoenician Ceramic Repertoire between the Levant and the West $9^{\text {th }}-6^{\text {th }}$ century BC. Proceedings of the International Conference held in Rome, 26 ${ }^{\text {th }}$ February 2010 (Quaderni di Archeologia Fenicio-Punica V), Roma 2010, pp. 211-253.

RENZI, M.

2007 Estudio tipológico y funcional de las toberas del yacimiento de la Fonteta (Guardamar del Segura, Alicante): Trabajos de Prehistoria 64 (2007), pp. 165-178.

SAÏDAH, R

$1966 \quad$ Fouilles de Khaldé. Rapport préliminaire sur la première et deuxième campagnes (19611962) (Bullettin du Musée de Beyrouth 19), Beyrouth 1966.

SANTOCCHINI GERG, S

in stampa Le importazioni etrusche nella Sardegna d'età arcaica: Nuovi dati e osservazioni preliminari: M.H. FANTAR - A. FERJAOUI (edd.), Actes du VII ${ }^{e ́ m e}$ Congrès International des Études Phéniciennes et Puniques, Hammamet 10-14 novembre 2009, in stampa.

SCHUBART, H. - NIEMEYER, H.G.

1976 Trayamar. Los hipogeos fenicios y el asentamiento en la desembocadura del río Algarrobo (Excavaciones Arqueológicas en España 90), Madrid 1976.

SECCI, R.

2006 La ceramica punica: E. Acquaro - C. Del VAis - A.C. FAriselli (a cura di), Beni culturali e antichità puniche: la necropoli meridionale di Tharros (Biblioteca di Byrsa 4; Tharrica 1), Sarzana 2006, pp. 173-186.

SECHI, E

2006 Il relitto di Sa Tonnara-A: A. MAstino - P.G. SPANU - R. ZuCCA, Tharros Felix 2, Roma 2006, pp. 146-180.

SPANU, P.G.

2006 Ricerche di archeologia subacquea nell'area del Korakodes portus: A. MAstiNo - P.G. SPANU - R. ZuCCA, Tharros Felix 2, Roma 2006, pp. 94-142.

SPARKES, B.A. - TALCOTT, L.

1970 Black and Plain Pottery of the 6th and 4th centuries B.C. (The Athenian Agora 12), Princeton 1970.

TEKKI, A.

2008 L'artisanat métallurgique punique à Carthage: l'exemple des ateliers de réduction du fer et du cuivre: J. GonZÁLEZ - P. RugGeri - C. VISMARA - R. ZuCCA (a cura di), L’Africa 
romana. Le ricchezze dell'Africa. Risorse, produzioni, scambi. Atti del XVII convegno di studio, Sevilla, 14-17 dicembre 2006 (Pubblicazioni del Centro di Studi Interdisciplinari sulle Province Romane dell’Università degli Studi di Sassari 35), Roma 2008, pp. 879892.

TRONCHETTI, C.

1988 Bithia II: La ceramica a vernice nera a pasta grigia: Quaderni della Soprintendenza Archeologica per le Province di Cagliari e Oristano 5 (1988), pp. 141-152.

1993 La ceramica attica a vernice nera: C. TRONCHETTI ET ALII, Lo scavo di Via Brenta a Cagliari. I livelli fenicio-punici e romani (Quaderni della Soprintendenza Archeologica per le Province di Cagliari e Oristano, Supplemento 9), Cagliari 1993, pp. 67-83.

1996 La ceramica della Sardegna romana, Milano 1996.

UBERTI, M.L.

2010 Tharros e i Sardi: A. FERJAOUI (a cura di), Carthage et les autochtones de son empire du temps de Zama. Hommage à Mhamed Hassine Fantar, Siliana-Tunis 10-13 mars 2004, Tunis 2010, pp. 155-160.

UNALI, A.

2010 Sulky: la ceramica attica a vernice nera: M. MiLANESE - P. RUGGERI - C. VISMARA (a cura di), L'Africa romana. I luoghi e le forme dei mestieri e della produzione nelle province africane. Atti del XVIII convegno di studio, Olbia, 11-14 dicembre 2008 (Pubblicazioni del Centro di Studi Interdisciplinari sulle province romane dell’Università degli Studi di Sassari 36), Roma 2010, pp. 1227-1240.

USAI, A.

1988 La stazione nuragica di Sa Serra (Serrenti - Cagliari): Quaderni della Soprintendenza Archeologica per le Province di Cagliari e Oristano 5 (1988), pp. 65-76.

VAN DOMMELEN, P. - GÓMEZ BELLARD, C. - PÉREZ JORDÀ, G.

2010 Produzione agraria nella Sardegna punica fra cereali e vino: M. MILANESE - P. RUGGERI C. Vismara (a cura di), L'Africa romana. I luoghi e le forme dei mestieri e della produzione nelle province africane. Atti del XVIII convegno di studio, Olbia, 11-14 dicembre 2008 (Pubblicazioni del Centro di Studi Interdisciplinari sulle province romane dell’Università degli Studi di Sassari 36), Roma 2010, pp. 1187-1202.

VEGAS, M.

1999 Phöniko-punische Keramik aus Karthago: F. RAKоB (Hrsg.), Die deutschen Ausgrabungen in Karthago III, Mainz am Rhein 1999, pp. 93-219.

Villada PARedes, F. - RAMON TORRES, J. - SuÁrez PAdilla, J.

2010 El asentamiento protohistórico de Ceuta. Indígenas y Fenicios en la orilla norteafricana del estrecho de Gibraltar, Ceuta 2010.

XELLA, P.

2010 Per un 'modello interpretativo' del tofet: il tofet come necropoli infantile?: G. BARTOLONI - P. Matthiae - L. Nigro - L. Romano (a cura di), Tiro, Cartagine, Lixus: nuove acquisizioni. Atti del Convegno Internazionale in onore di Maria Giulia Amadasi Guzzo, Roma 24-25 novembre 2008 (Quaderni di Vicino Oriente IV), Roma 2010, pp. 259-279. 


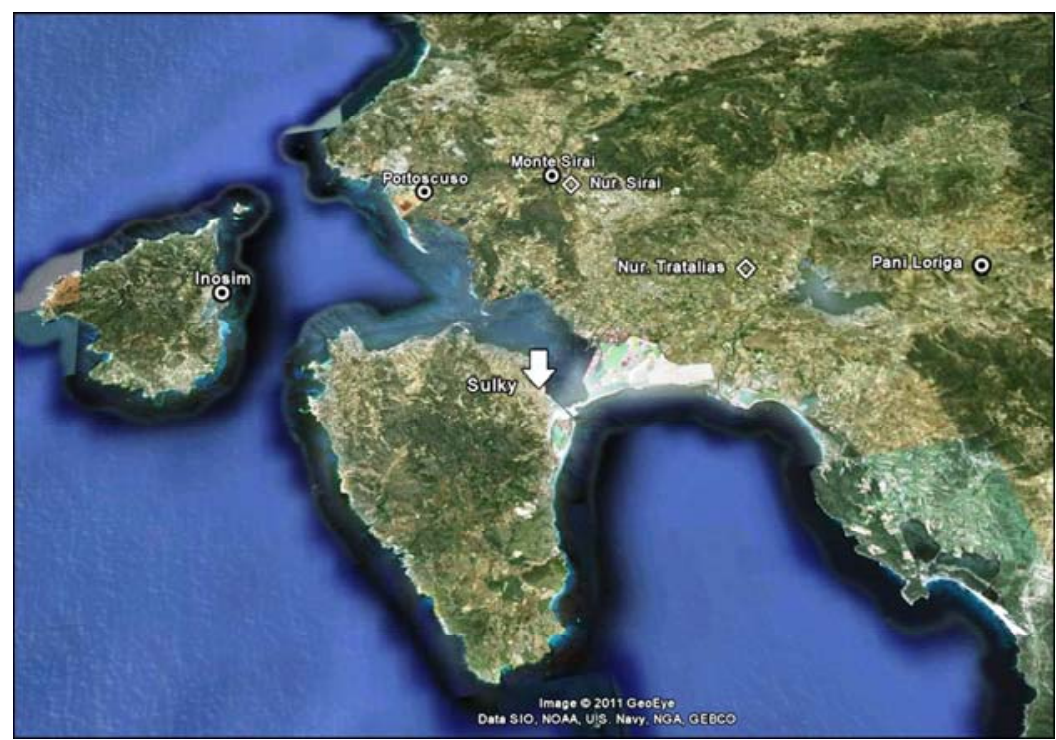

Fig. 1: Veduta satellitare della Sardegna sud-occidentale con indicazione dei principali insediamenti (rielaborazione da Google Earth).

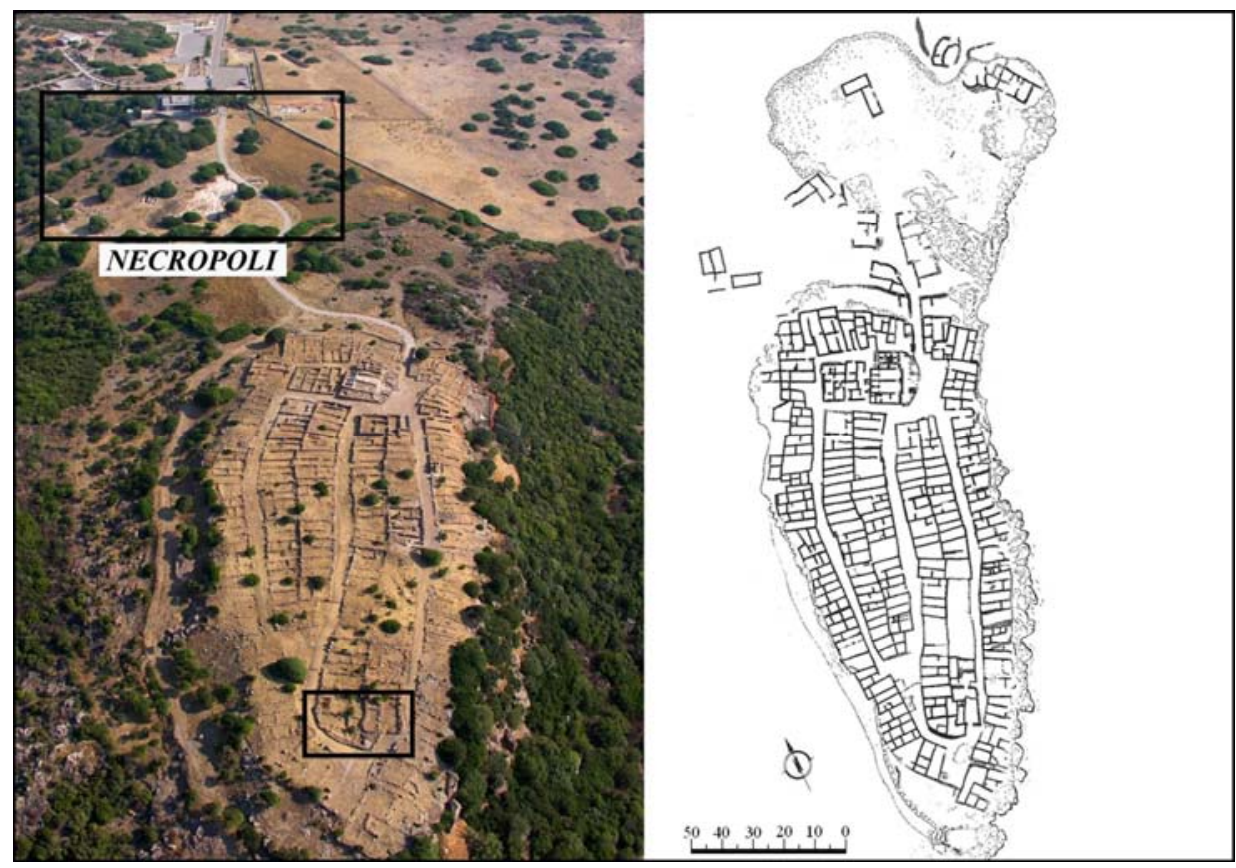

Fig. 2: Veduta aerea dell'insediamento e pianta generale dell'abitato di Monte Sirai. 


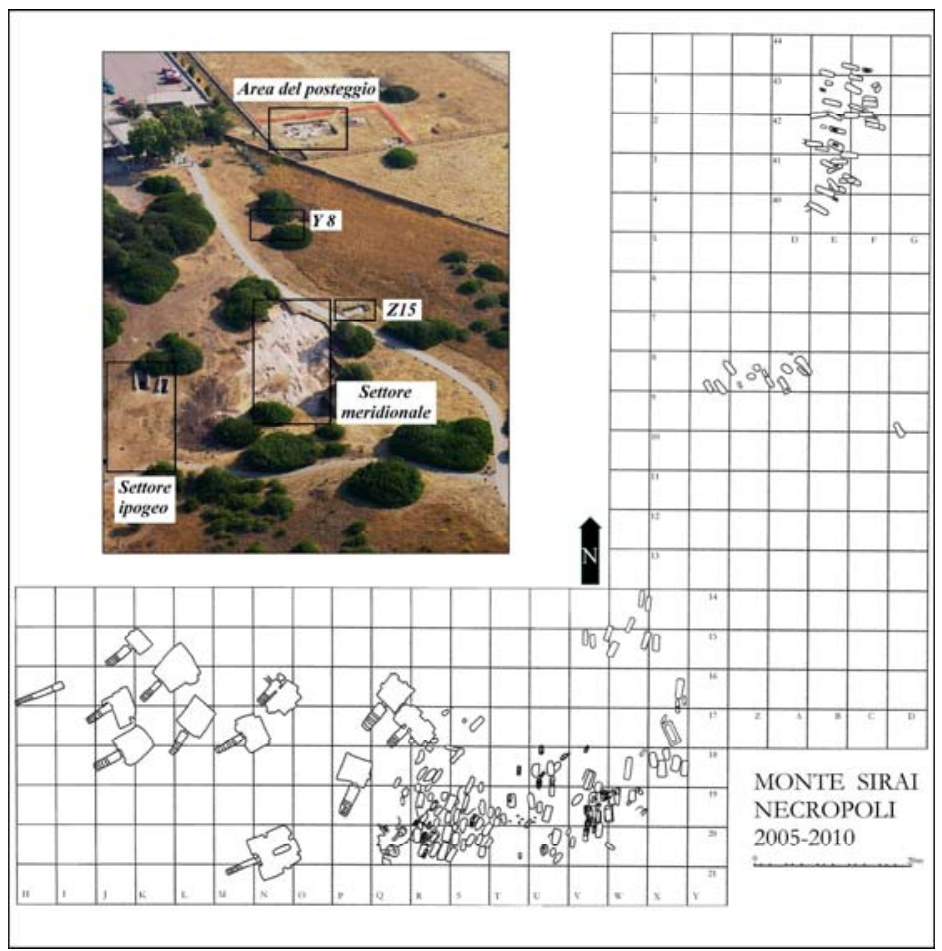

Fig. 3: Veduta aerea e planimetria complessiva della necropoli fenicia e punica di Monte Sirai (elaborazione di R. Pla Orquín).

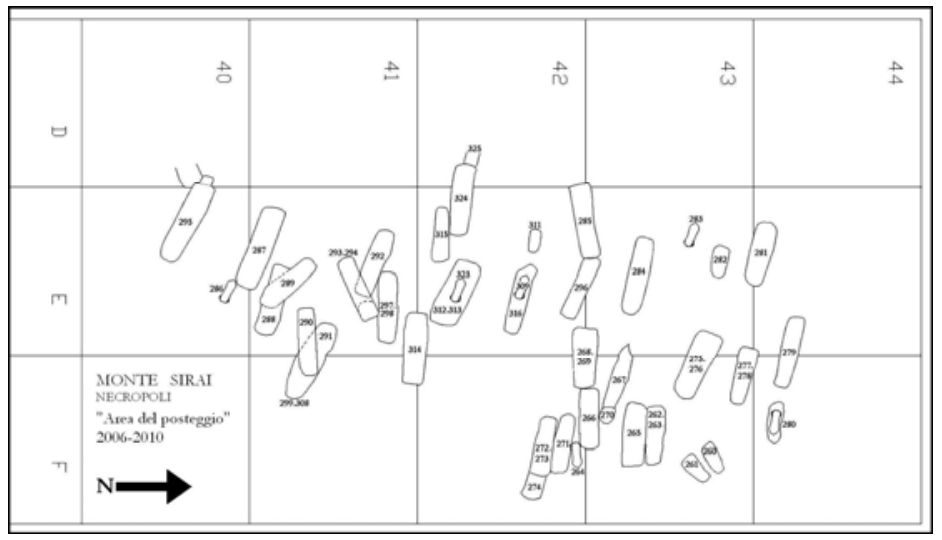

Fig. 4: Planimetria di dettaglio del settore settentrionale della necropoli denominato "area del posteggio” (elaborazione di R. Pla Orquín). 


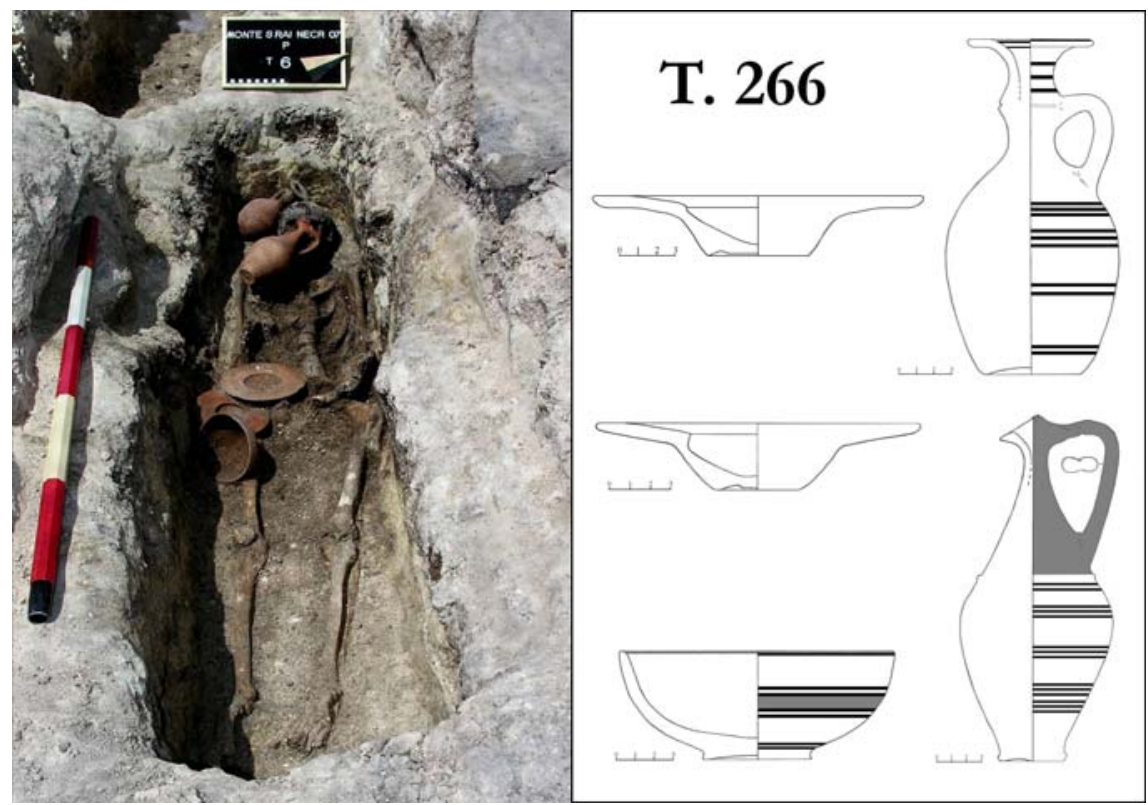

Fig. 5: Contesto della T. 266 in corso di scavo e relativo corredo.

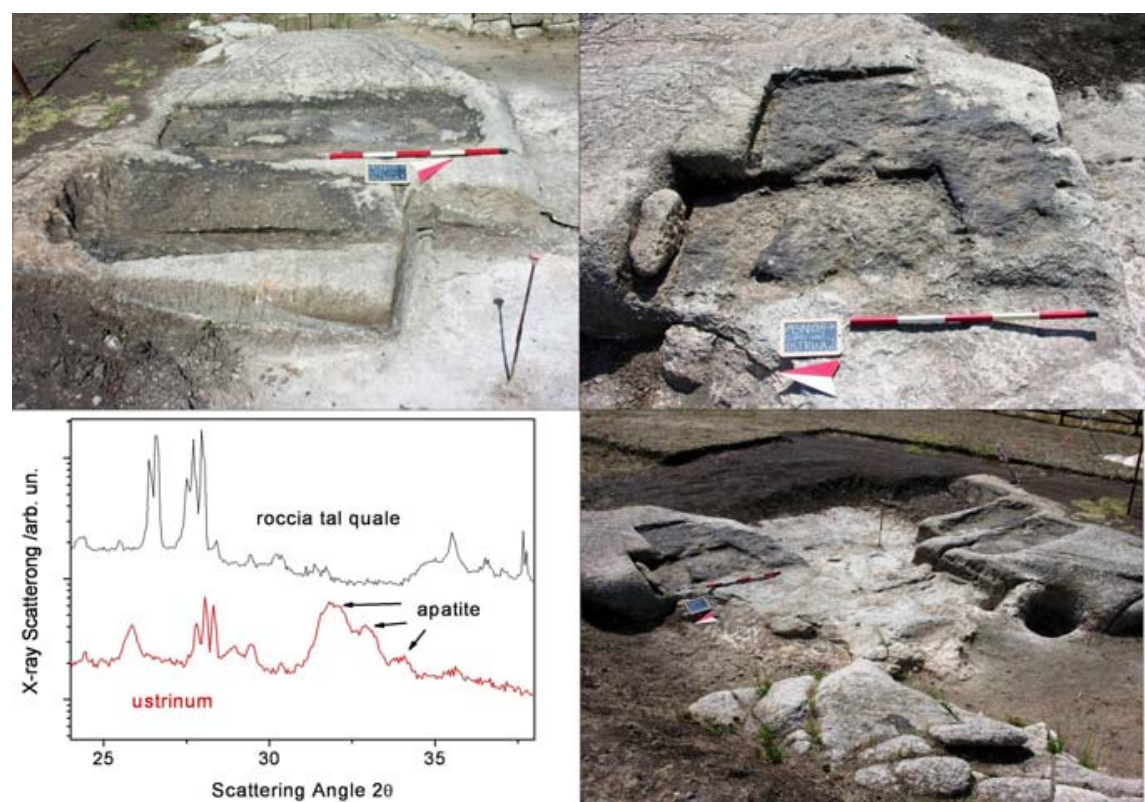

Fig. 6: Fotografie degli ustrina e diagramma di diffrazione ai raggi $\mathrm{X}$ dei campioni analizzati. 


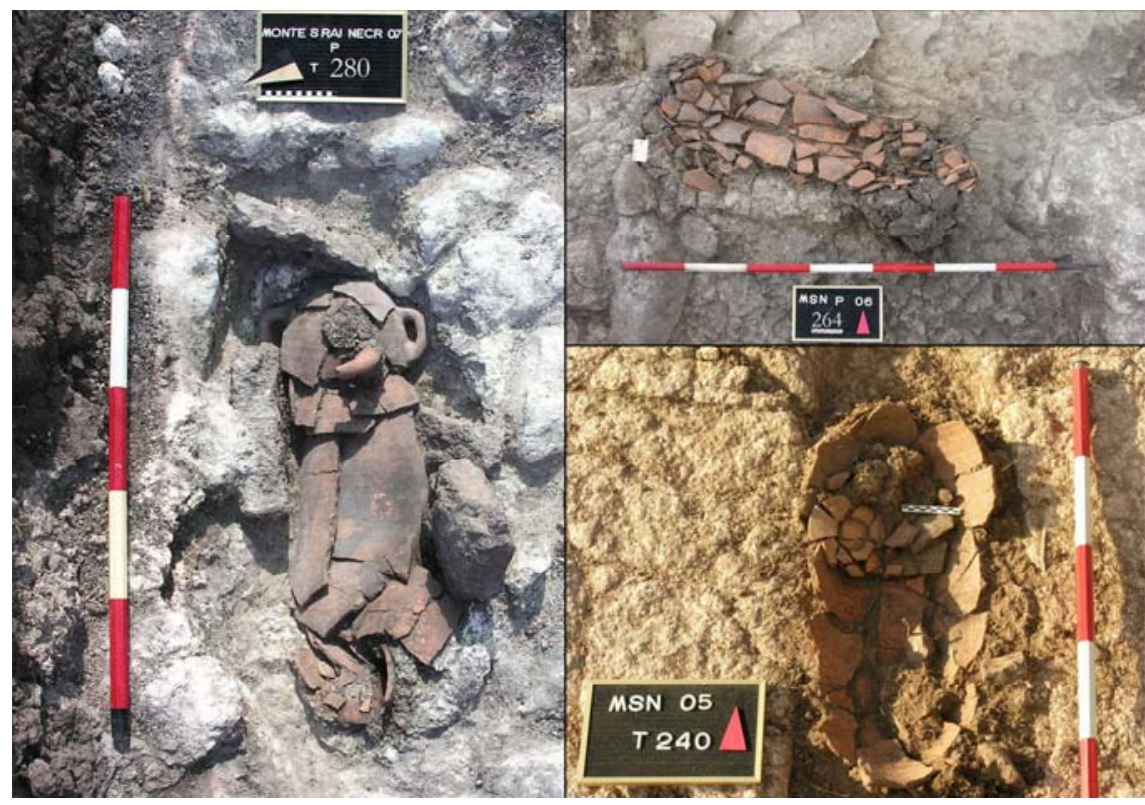

Fig. 7: Fotografie degli enchytrismoi TT. 240, 264, 280 in corso di scavo.
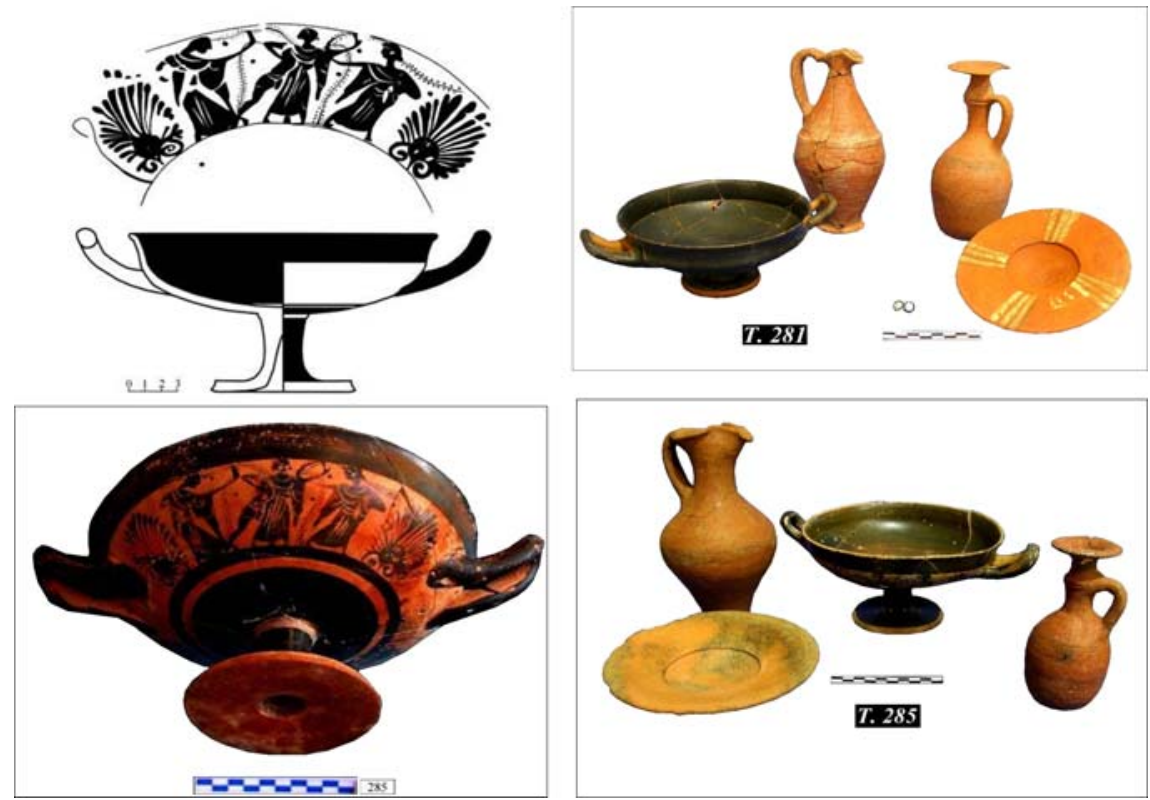

Fig. 8: Immagini relative ai corredi delle TT. 281 e 285 con importazioni attiche. 


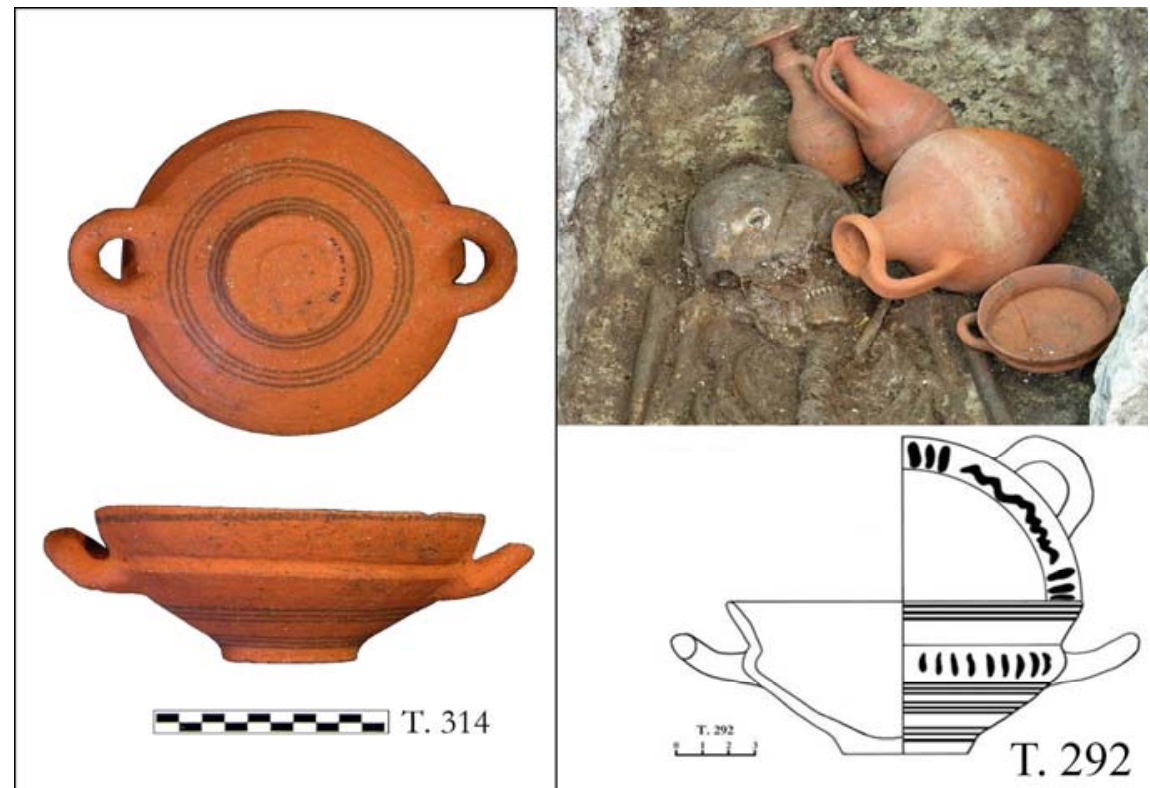

Fig. 9: Coppe biansate dai contesti delle T. 292 e 314.

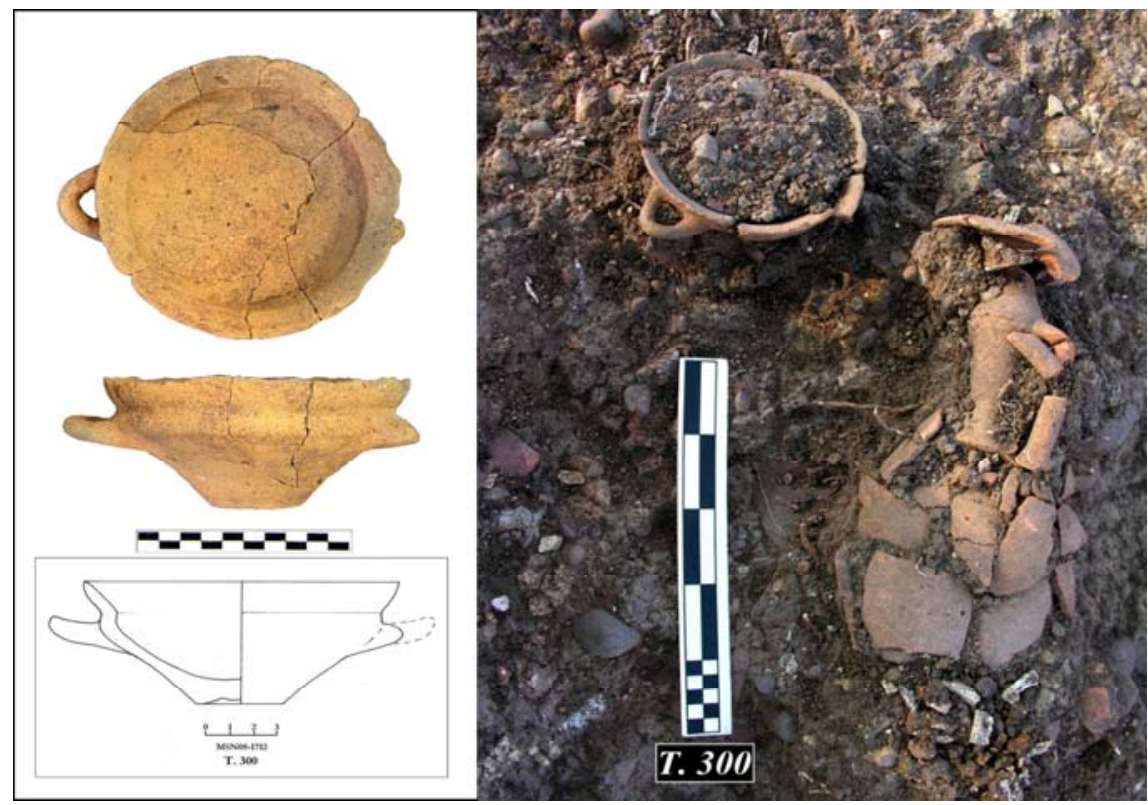

Fig. 10: Contesto in corso di scavo della T. 300 e coppa biansata dal corredo. 


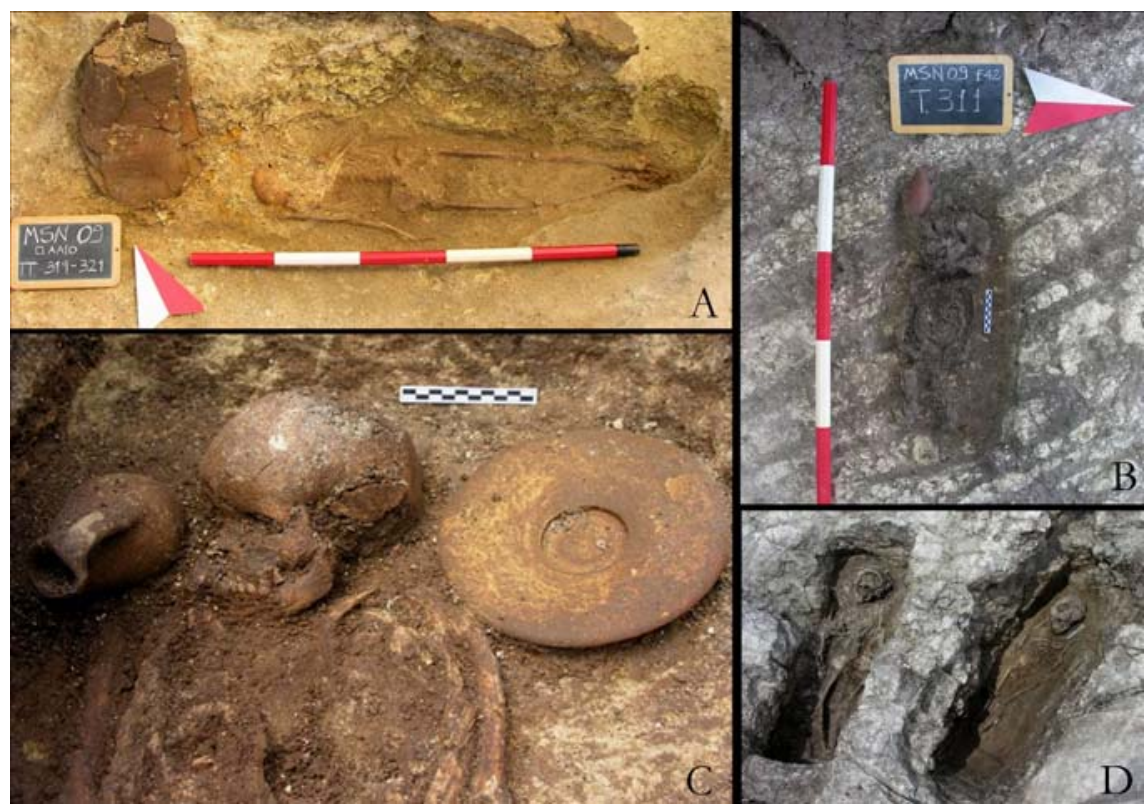

Fig. 11: Immagini relative a deposizioni infantili della necropoli (A: T. 321; B: T. 311; C: T. 315; D: TT. 260, 261).

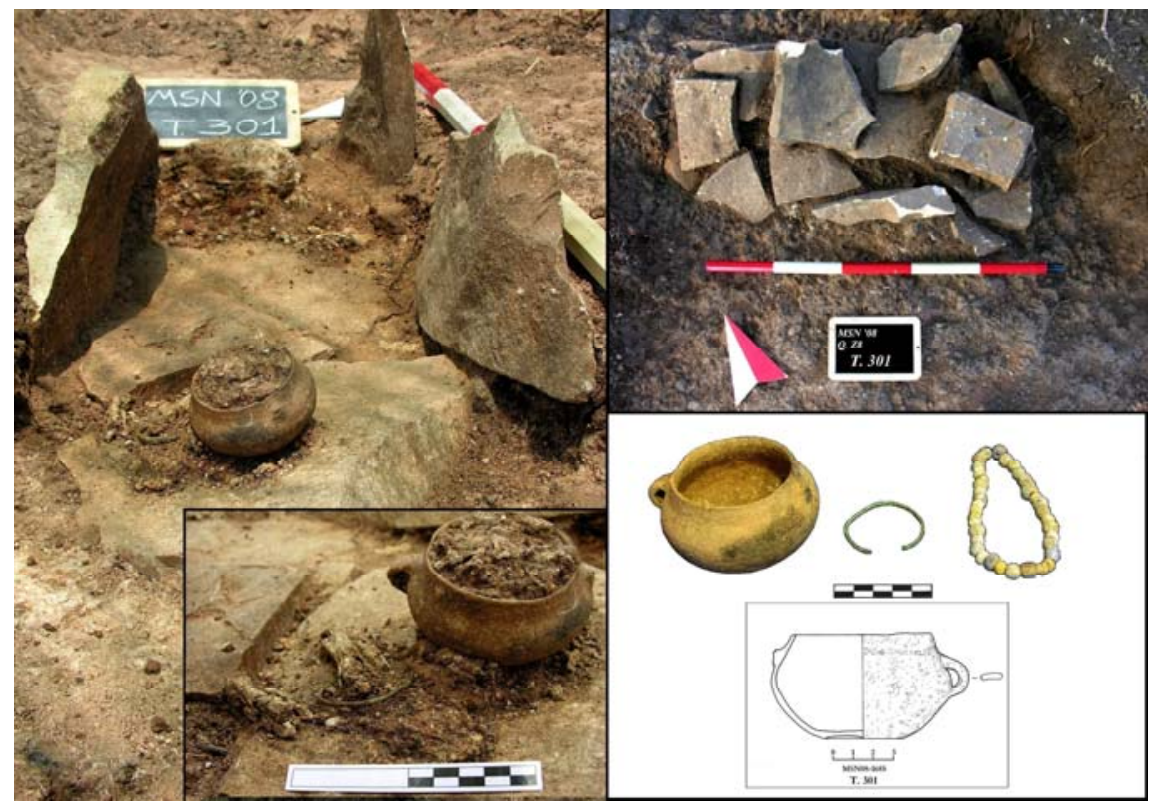

Fig. 12: Immagini relative al contesto della deposizione infantile T. 301. 


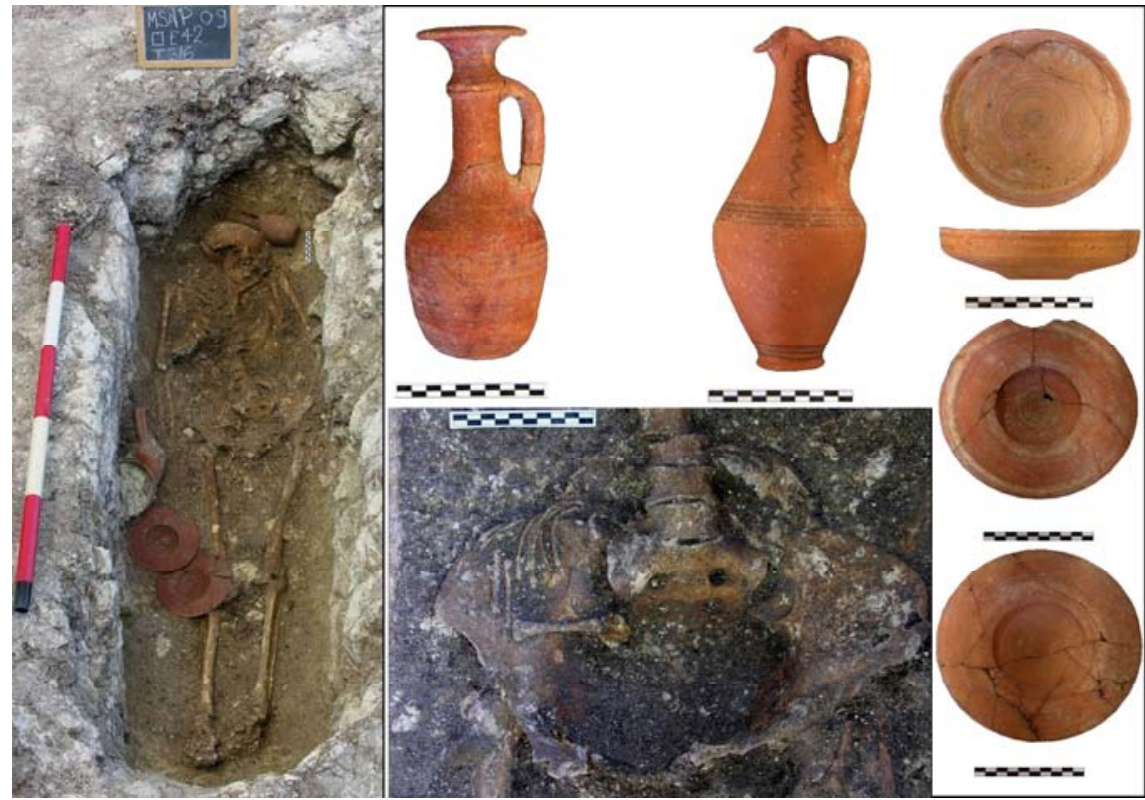

Fig. 13: Immagini relative al contesto della deposizione femminile T. 316.

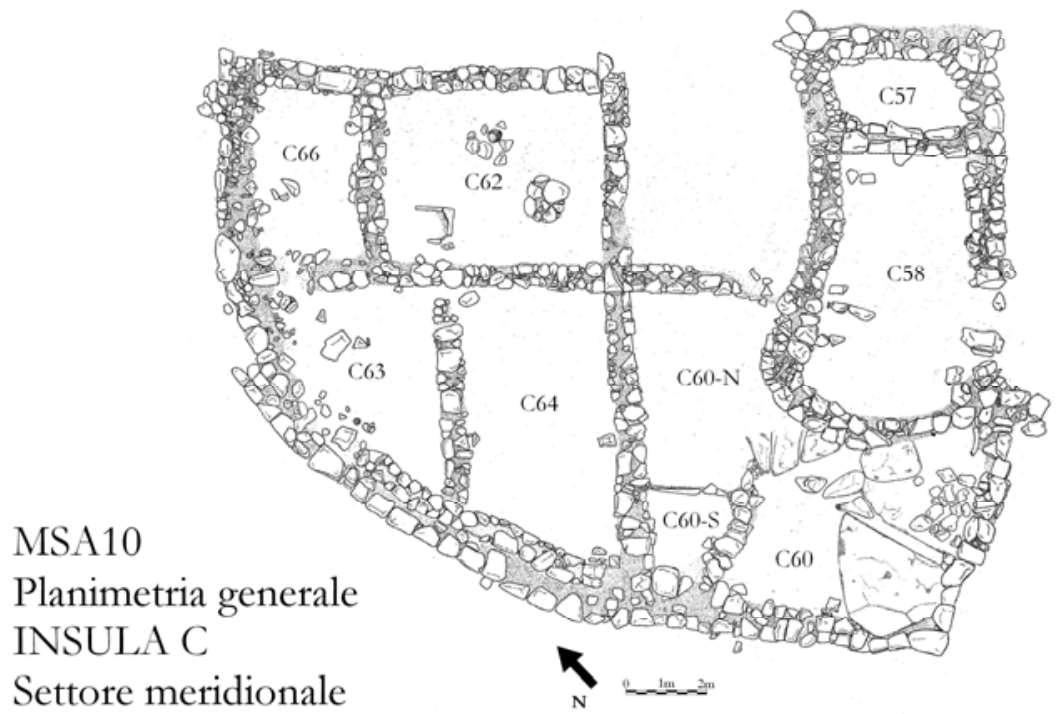

Fig. 14: Planimetria complessiva del settore meridionale dell'Insula C (C-Sud). 


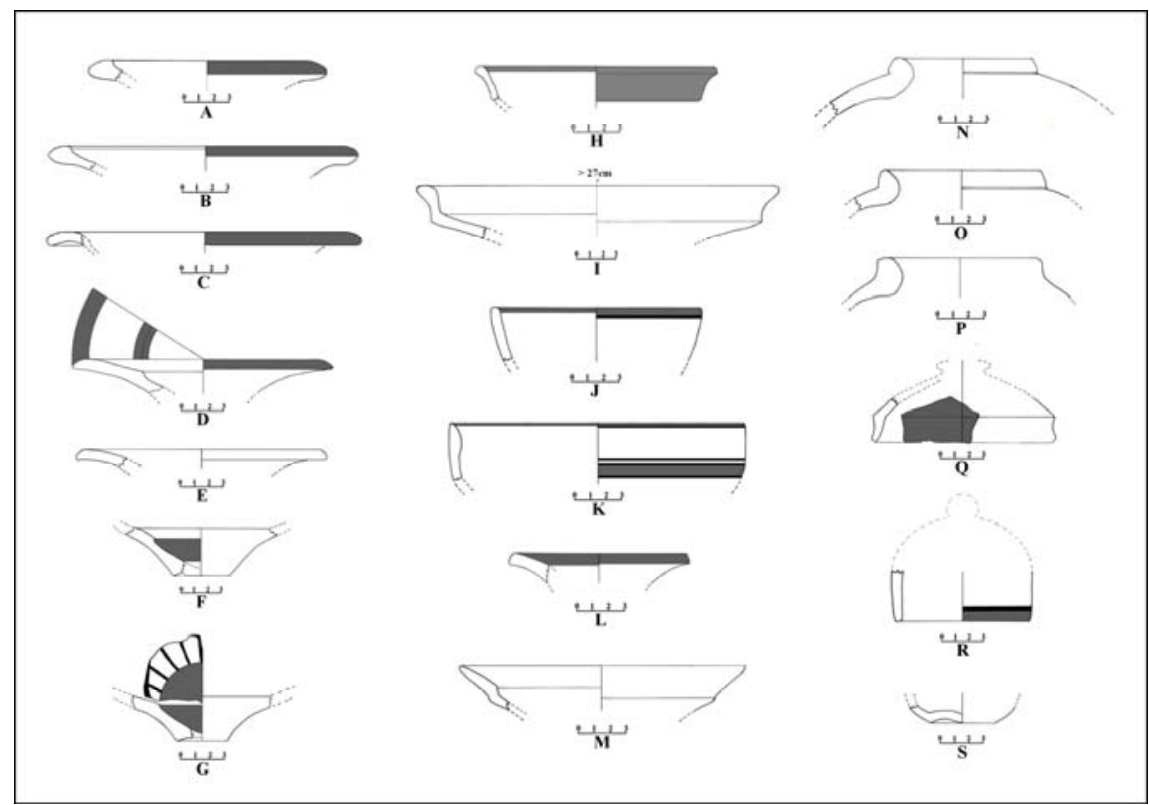

Fig. 15: Selezione di forme aperte, anfore, coperchi e brocche di età arcaica (730-500 a.C.).

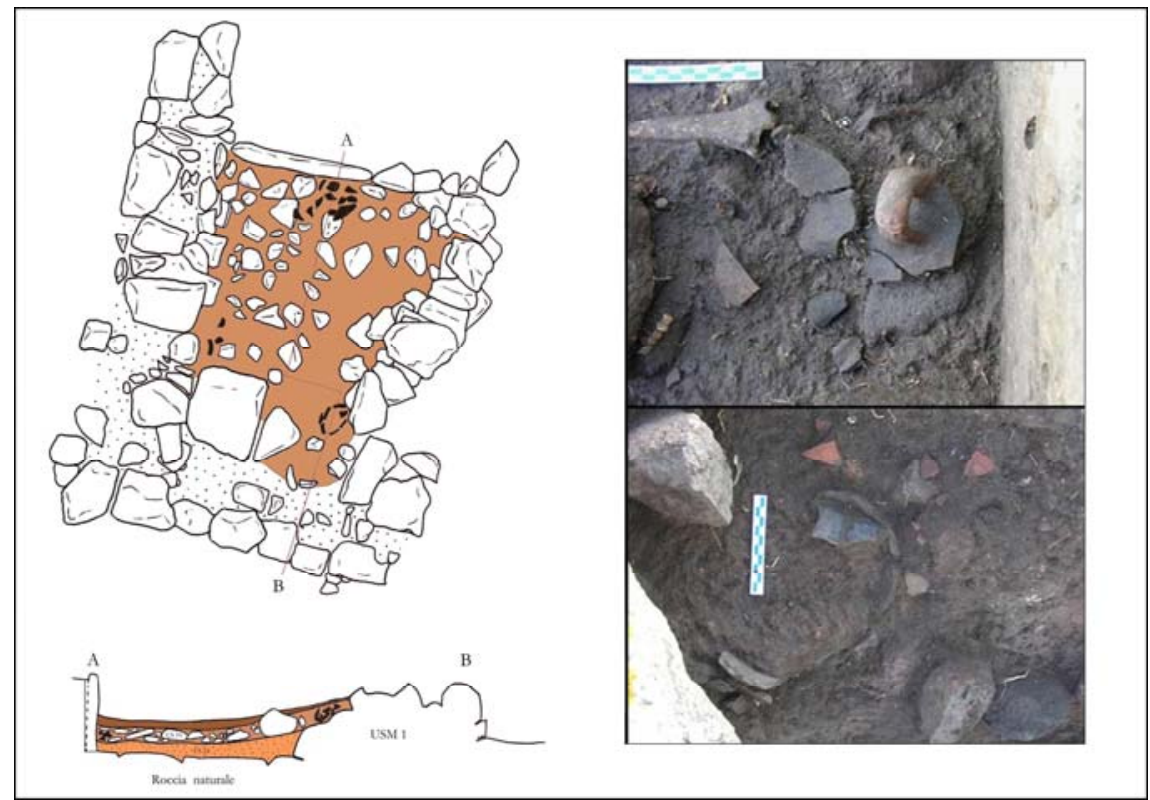

Fig. 16: Planimetria e sezione del vano C60S con immagini delle pentole d'impasto in corso di scavo. 


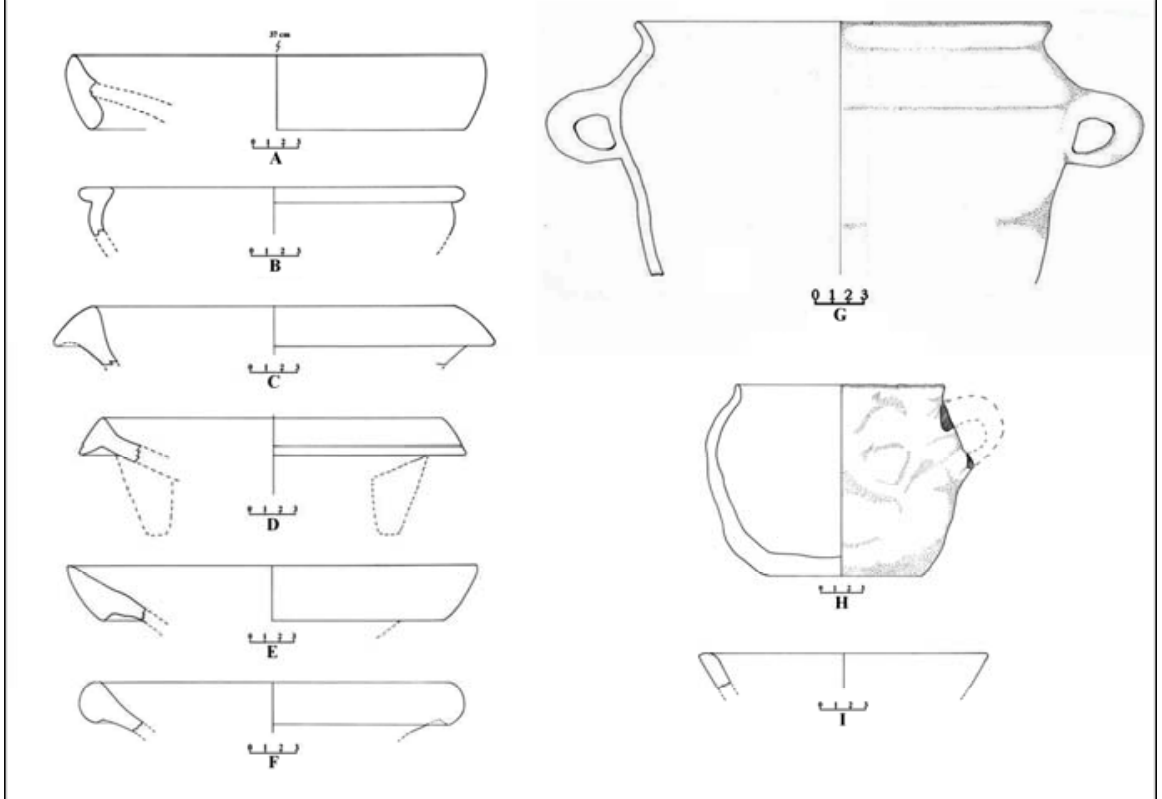

Fig. 17: Bacini, tripodi e forme d'impasto di età arcaica (730-500 a.C.).

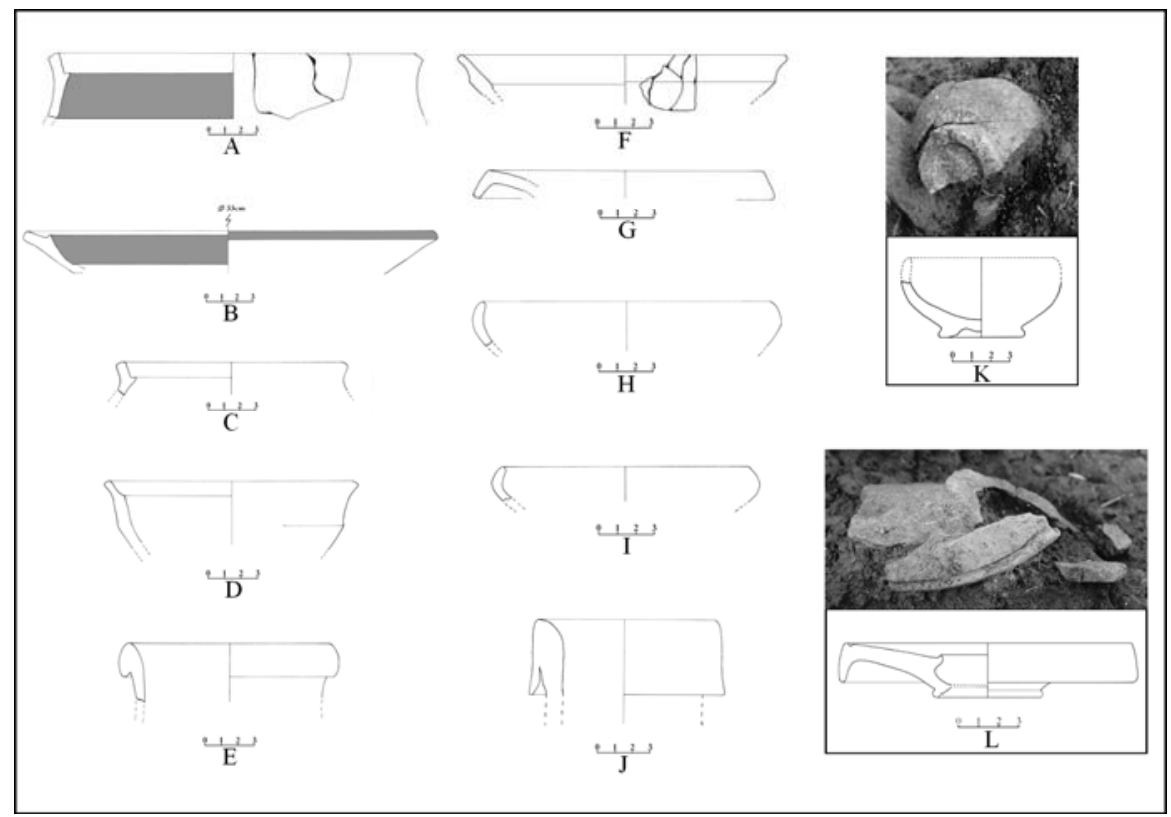

Fig. 18: Selezione di forme ceramiche di età punica ed ellenistica (V-I sec. a.C.). 